\title{
Telychian (Llandovery, Silurian) conodonts from the LaPorte City Formation of eastern lowa, USA (East-Central lowa Basin) and their implications for global Telychian conodont biostratigraphic correlation
}

\author{
Christopher B.T. Waid and Bradley D. Cramer
}

\begin{abstract}
Conodonts from the LaPorte City Formation of eastern lowa (East-Central lowa Basin) indicate an early to middle Telychian age for the formation. Conodonts diagnostic of the Pterospathodus eopennatus Superzone, Pterospathodus eopennatus ssp. $\mathrm{n}$. 2 Zone, and Pterospathodus amorphognathoides angulatus Zone were recovered, allowing for the first direct comparison of the stratigraphic ranges of conodont species in the Illinois and Baltic Basins. A heretofore undescribed species of Pseudolonchodina similar to Pseudolonchodina fluegeli occurs in the LaPorte City Formation. It is distinguished from Pseudolonchodina fluegeli by the absence of fused denticles on $\mathrm{Pa}$ elements and discrete to nearly-discrete denticles on the other elements. Due to the fragmented nature of the specimens, the species is left in open nomenclature. Wurmiella? polinclinata polinclinata ranges much lower in the East-Central lowa Basin (Pt. eopennatus ssp. n. 2 Zone) than the Baltic Basin, and therefore cannot be used as an index fossil diagnostic of the Pt. am. amorphognathoides Zone in global correlations. This study documents the utility of the small limestone formations on the northwest flank of the East-Central lowa Basin for refining global Silurian conodont biostratigraphic zonation.
\end{abstract}

Christopher B.T. Waid. Department of Earth and Environmental Sciences, University of lowa, 115 Trowbridge Hall, lowa City, IA 52242, USA; Present address: Ohio Geological Survey, 2045 Morse Rd. Building C, Columbus, OH 43229, USA. christopher.waid@dnr.ohio.gov Bradley D. Cramer. Department of Earth and Environmental Sciences, University of lowa, 115 Trowbridge Hall, lowa City, IA 52242, USA. bradley-cramer@uiowa.edu

Keywords: Conodonts; Biostratigraphy; Silurian; Llandovery; North American Midcontinent; LaPorte City Formation

Submission: 20 May 2016 Acceptance: 13 July 2017

\footnotetext{
Waid, Christopher B.T., and Cramer, Bradley D. 2017. Telychian (Llandovery, Silurian) conodonts from the LaPorte City Formation of eastern lowa, USA (East-Central lowa Basin) and their implications for global Telychian conodont biostratigraphic correlation. Palaeontologia Electronica 20.2.39A: 1-37

palaeo-electronica.org/content/2017/1950-telychian-conodonts-from-iowa

Copyright: August 2017 Paleontology Society. This is an open access article distributed under the terms of Attribution-NonCommercial-ShareAlike 4.0 International (CC BY-NC-SA 4.0), which permits users to copy and redistribute the material in any medium or format, provided it is not used for commercial purposes and the original author and source are credited, with indications if any changes are made.

creativecommons.org/licenses/by-nc-sa/4.0/
} 
The order that new species of conodonts appear in the rock record can be used to determine their relative age, and rocks that contain the same fossil were likely deposited at similar times. The order that Silurian conodont species appear and disappear is well understood in the Baltic Basin, but there has been very little conodont data from North America that is directly comparable to the Baltic. The Telychian (Silurian) Age conodont data from this study is the first from North America that is directly comparable to data from the Baltic Basin and allows for the most precise relative age assignments to date for the Silurian strata of lowa. They also indicate that origination and extinction of several species occurred at different times in North America than in the Baltic.

\section{INTRODUCTION}

The Silurian used to be considered an interval in earth history characterized by a stable, warm climate with high sea-level - a brief time of quiescence between Caledonian and Appalachian tectonism, continental-scale glaciations, and extinction intervals that characterized much of the Ordovician and Devonian periods. However, global application of high-resolution integrated biochemostratigraphic studies have shown that the amelioration of the Early Paleozoic Ice Age continued well into the Silurian, and that the Silurian Period was one of exceptional global biotic and climatic variability (Calner, 2008; Munnecke et al., 2010; Cooper et al., 2014). In particular, the global carbon cycle was more unstable during the Silurian than any other interval during the Phanerozoic Eon, with at least seven globally identified $\delta^{13} \mathrm{C}$ isotope excursions occurring over the approximate 24.6 Myr duration of the period (Munnecke et al., 2010; Cramer et al., 2011; Melchin et al., 2012). These combined instabilities in the ocean-atmospherebiosphere system during the Silurian make it an ideal interval to study the complex interactions and interdependencies of climate, the carbon cycle, and the biosphere.

To study cause-and-effect relationships between the numerous Earth-system cycles on the finest scales, the sedimentary rock record must be subdivided into the smallest chronostratigraphic units that can be demonstrated to be globally meaningful. Biostratigraphy-the application of the Law of Faunal Succession to correlate strata-is the framework upon which the relative timescale is built. Most biozonations rely on the first or last occurrence of a species in the rock record to define the boundary between biozones. In the past, these events were often considered geologically instantaneous, because chronostratigraphic resolution was rarely much finer than stage level (Cramer et al., 2015). However, recent advances in integrating numerous chronostratigraphic techniques demonstrate that differences in the timing of the first or last occurrence of a species in different paleobasins can be resolved (e.g., Cody et al., 2008; Cramer et al., 2010). The diachroneity of biostratigraphically useful species in the rock record needs to be understood and quantified before accurate correlation of paleoclimate proxies can be achieved. The LaPorte City Formation of lowa offers an opportunity to compare the stratigraphic ranges of conodonts in the Telychian Stage to those of more thoroughly studied regions, such as Baltica.

\section{Global Telychian Conodont Zonation}

Conodonts are one of the primary fossils used for biostratigraphic correlation of Silurian marine strata. The highest-resolution Silurian conodont zonations were developed from numerous cores and outcrops of strata from the Telychian to Ludfordian stages (Llandovery-Ludlow series) from the Baltic Basin (Jeppsson, 1997; Männik, 1998; Jeppsson and Calner, 2002; Calner and Jeppsson, 2003; Jeppsson et al., 2006; Männik 2007a, 2007b). The Telychian zonation primarily relies on first occurrences of the evolutionarily-related subspecies of the genus Pterospathodus (Figure 1). The nominal subspecies of these zones, Pterospathodus eopennatus ssp. n. 1 and ssp. n. 2 sensu Männik (1998), Pt. amorphognathoides angulatus, Pt. am. lennarti, Pt. am. lithuanicus, and Pt. am. amorphognathoides were formally recognized and defined in Männik (1998), so Telychian conodont studies and zonations prior to the recognition of these subspecies used other less precise, and often regional conodont biozones (e.g., Walliser, 1964; Aldridge, 1972; Over and Chatterton, 1987).

Because of their precision, the Telychian zones and superzones of Männik (2007a) were adopted as a global zonation scheme (Cramer et al., 2011), and were refined by graphic correlation techniques by Kleffner and Barrick (2010). Conodonts associated with the superzones can be found worldwide, and most of the zones can be consistently resolved in widespread paleobasins (Männik, 2007a). Männik (2007a) was able to reliably distinguish several subzones in the Baltic, mostly using the last occurrences of short-ranged species within the Pterospathodus-defined zones 


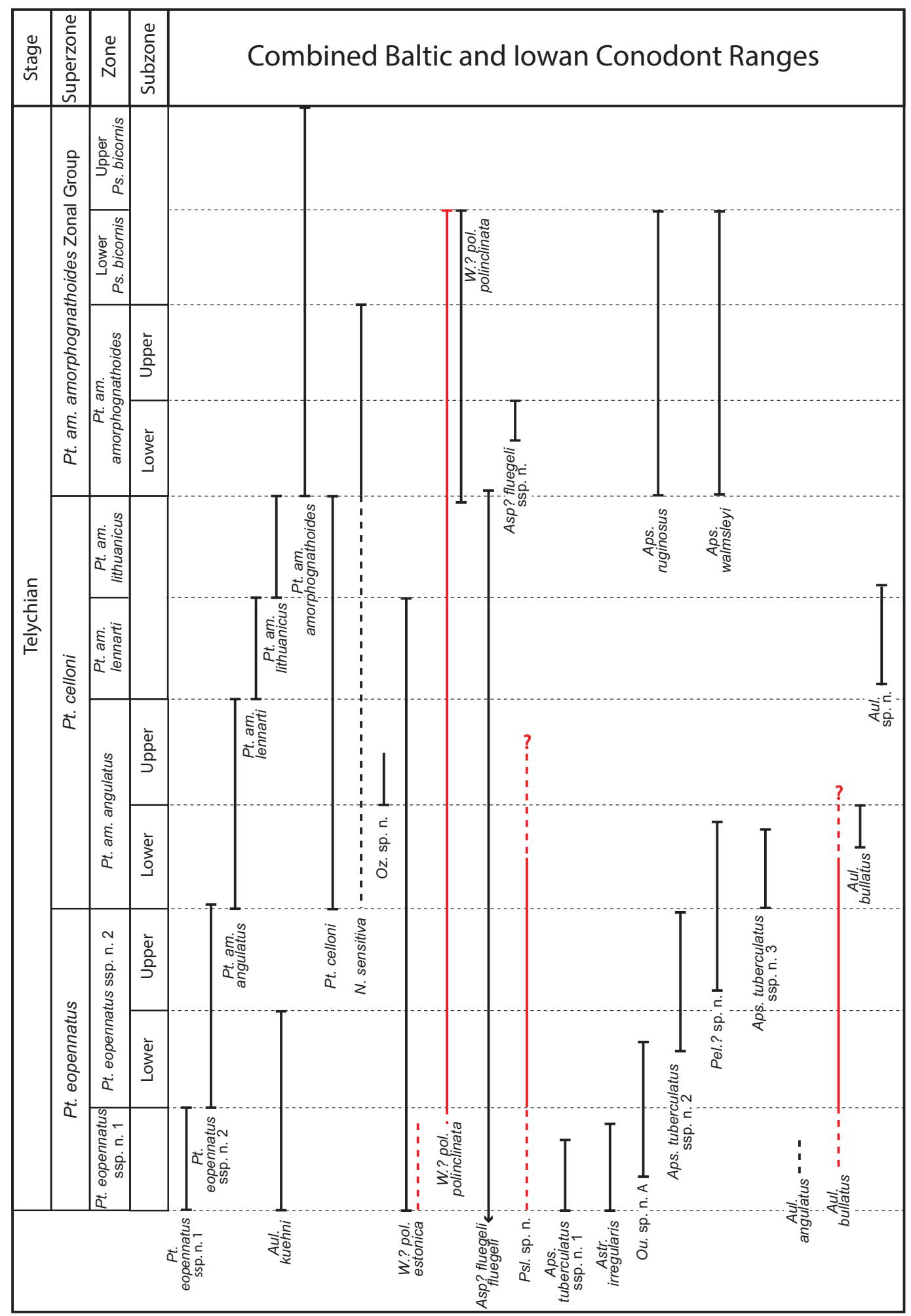

FIGURE 1. Telychian conodont zonation based on Baltic fauna with ranges of biostratigraphically important species. Red lines are used for ranges in lowa when they differ from the Baltic. Wurmiella? polinclinata polinclinata and Aulacognathus bullatus extend lower in lowa than in the Baltic. Wurmiella? polinclinata estonica does not range as high in lowa. Dashed lines in Pteropathodus eopennatus ssp. n. 1 Zone indicate uncertainty in stratigraphic range in lowa. Modified from Männik (2007a). 
(Figure 1). Unfortunately, the global stratigraphic ranges of many of the species used to define the subzones, and other species that are associated with particular zones in the Baltic, are unclear at the present. To test the robustness and global utility of these subzones, conodont biostratigraphic data from geographically widespread paleobasins must be compared with those from the Baltic.

\section{Laurentian Telychian Conodont Zonation}

In Laurentia, most Silurian conodont research was done prior to the taxonomic revisions of Pterospathodus. This means that the stratigraphic ranges of Telychian conodont species are understood using a different, and less precise, biostratigraphic framework than those from the Baltic (Kleffner, in McLaughlin et al., 2008). To further complicate matters, many of the "classical" North American Silurian successions in the Appalachian Foreland Basin were punctuated by intervals of heavy clastic input from the erosion of the Taconic Highlands (Brett et al., 1998), making recovery of well-preserved conodont faunas and accurate determination of stratigraphic ranges difficult. Carbonate deposition dominated other well-studied regions farther away from the Taconic Highlands, such as the Cincinnati Arch, Michigan Basin, and Illinois Basin, but most of the strata in those regions were subsequently dolomitized. The relative resistance of dolomite to breakdown in acid leads to poor conodont yields throughout most of these carbonate-rich successions. The spotty conodont ranges from the Telychian strata of the midcontinent basins of North America, and the use of outdated conodont taxonomy heretofore prevented high-resolution comparison with Baltic conodont faunas.

There are several understudied Silurian limestone formations and outliers in the northeastern and east-central part of lowa that hold potential for producing high-resolution conodont biozonations. One of the challenges with studying these units is that they often represent relatively short windows of time and are often geographically separated from other isolated limestone units, such that regional correlation with the major dolostone carbonates of the basin have been problematic. However, they can provide valuable conodont biostratigraphic data for refining global conodont zonations. The diverse and cosmopolitan conodont fauna documented in this study from the LaPorte City Formation (LPC) of eastern lowa allows for the first high-resolution conodont biostratigraphic study from the North American Midcontinent that is directly comparable to the zonation of Männik (2007a).

\section{Geologic Background}

The Silurian strata of lowa were deposited along a structural sag that connects the Illinois Basin with the North Kansas Basin (Witzke, 1992; Figure 2). The Illinois Basin formed in an extensional system related to the formation of the Late Proterozoic Reelfoot Rift (Macke, 1995). After the rift failed, thermal subsidence of the weakened crust provided accommodation space for sediments from the latest Proterozoic through the Pennsylvanian (Macke, 1995). The stable thermal subsidence of the basin, the stranding of clastics by high sea level, and tropical location allowed for carbonate-dominated deposition throughout the Silurian (Johnson, 1987; Torsvik et al., 1996).

The LPC is the lateral equivalent of parts of the Hopkinton Formation and the Buck Creek Quarry Member (BCQ) of the Scotch Grove Formation (Figure 3). It has the same general lithological characteristics of the Hopkinton Formation and the $B C Q$, but is considered to be a distinct formation because it was not dolomitized. The terms for the members of the Hopkinton and Scotch Grove formations will be applied to the LaPorte City within this study, because the lithological characteristics distinguishing them are present. The dolomitization process of the LPC was apparently halted by the influx of meteoric fresh water during subaerial exposure during the sea-level lowstand at the Silurian/ Devonian boundary (Witzke, 1992). See Witzke $(1981,1992)$ for detailed lithostratigraphy, regional correlation, and description of the macrofaunas of these units.

\section{STUDIED SECTIONS}

The LPC was collected from two localities in eastern lowa. The main section used for this study was the Garrison Core (GC), which was drilled in central Benton County, lowa. An outcrop section was also studied on a roadcut on road DX5, west of the town of Delhi in Delaware County (Figure 2). Previous conodont data from both locations was reported in Metzger (2005). Our data were able to be integrated with all of Metzger's (2005) data from the GC. Some of the DX5 section Metzger sampled was slumped over, so only data above the base of the LPC from Metzger (2005) are integrated with ours. Zonally significant specimens that were recorded but not illustrated in Metzger (2005) were photographed (Figure 4). Some of the species 


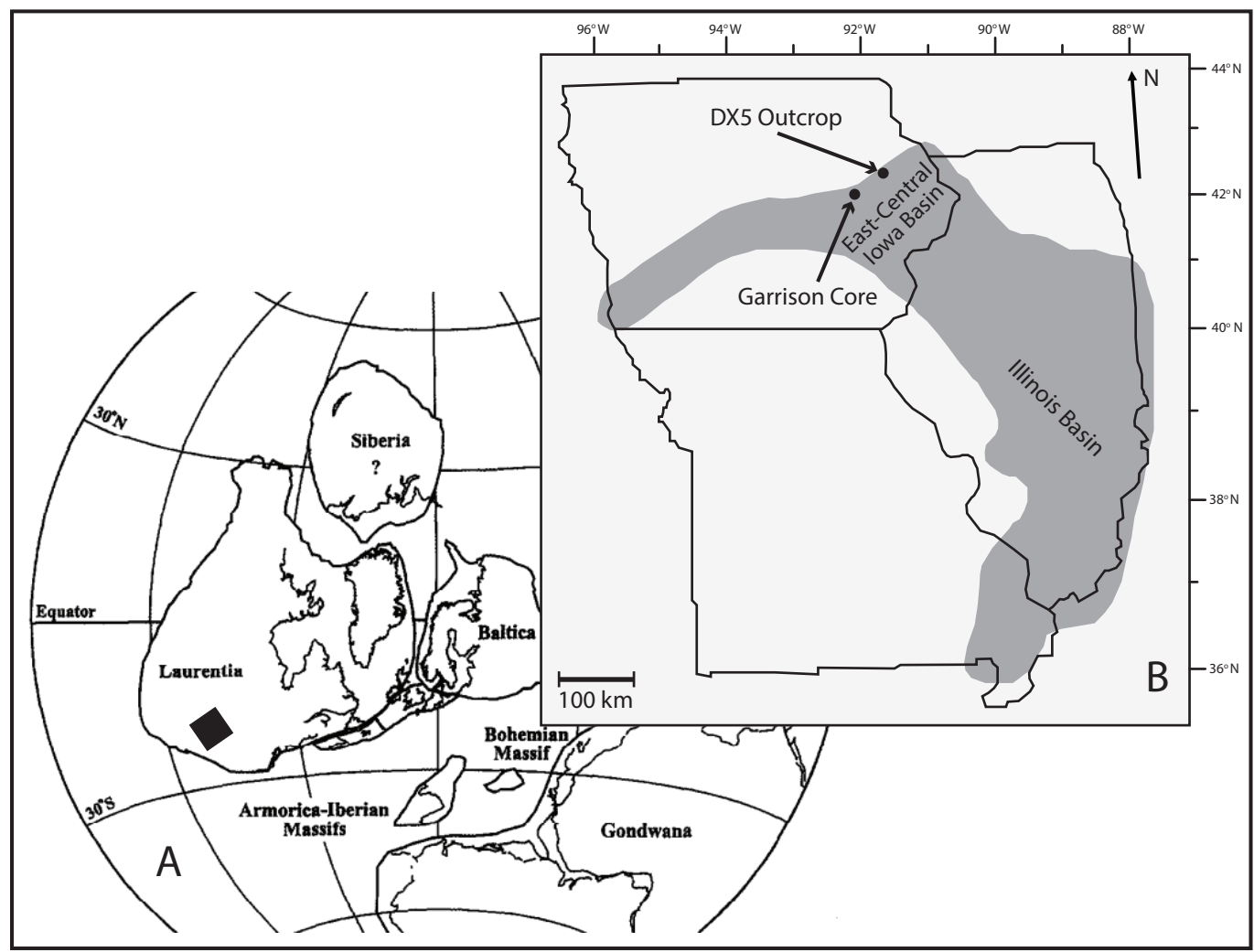

FIGURE 2. 1, Paleogeographic map showing the locations of the continents during the lower to middle Silurian. Black box indicates approximate location of the Illinois Basin. From Torsvik et al. (1996, figure 13). 2, Map showing the deepest part of the Illinois and East-Central lowa basins, and trans-lowa sag in lowa and nearby states during the Silurian. Shading indicates extent of basins. Modified from Cramer et al. (2006, figure 1).

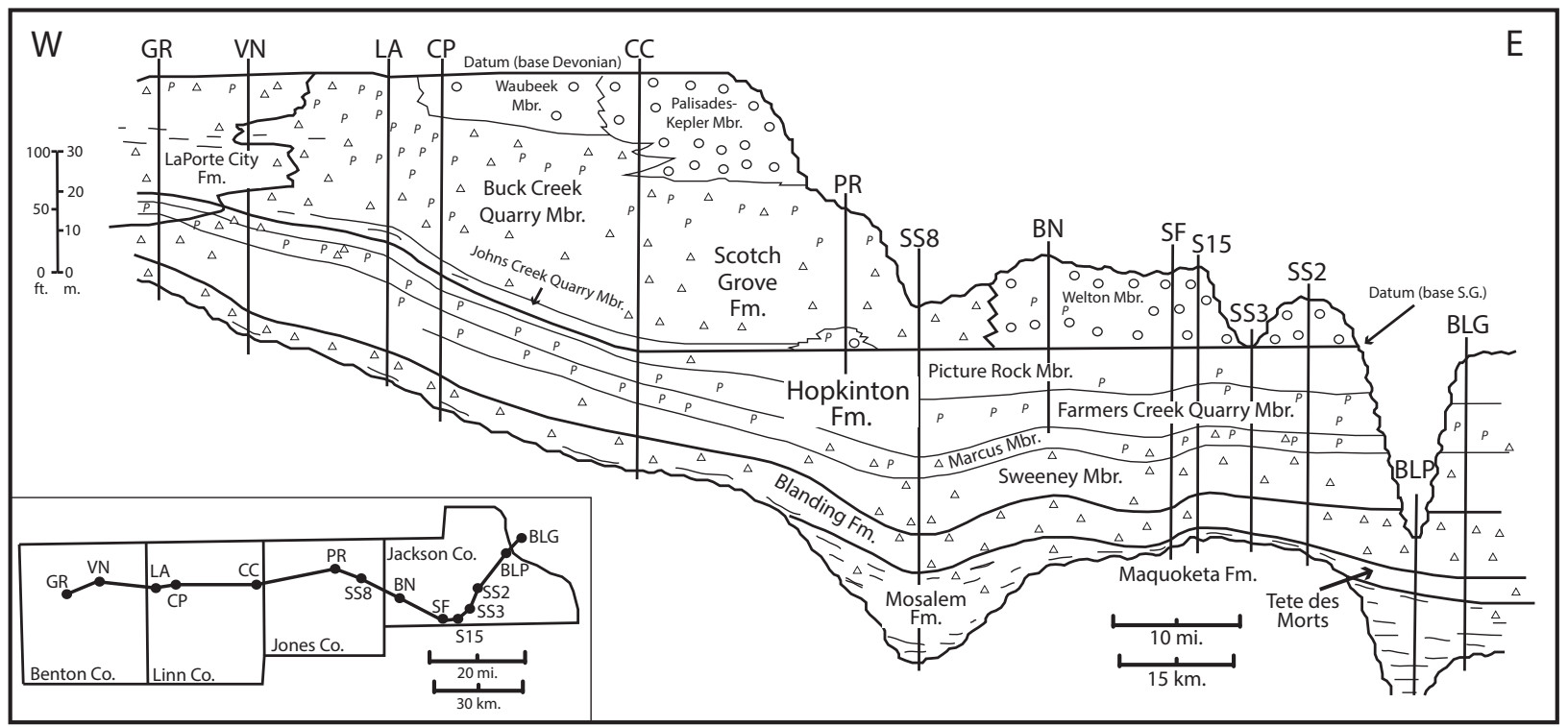

FIGURE 3. Cross section of Silurian strata in eastern lowa. The lower two members of the Hopkinton Formation become indistinguishable in the western part of the cross section. The Sweeney and Marcus members are referred to as Hopkinton A, the Farmers Creek Quarry Member as Hopkinton B, and the Picture Rock Member as Hopkinton $\mathrm{C}$ in this manuscript. Note the change in datum at the CC core in Linn County. Modified from Witzke (1992, figure 6). 


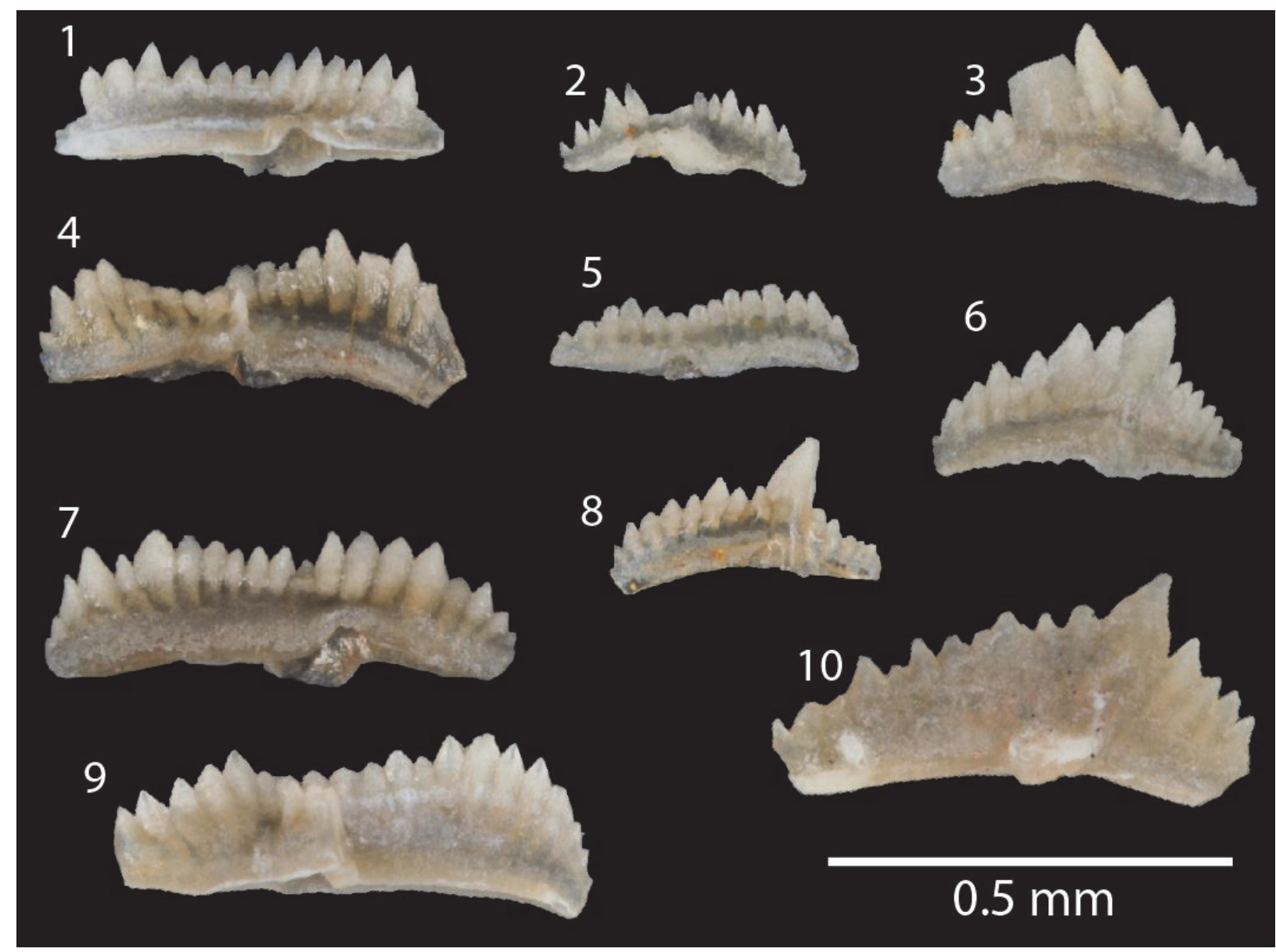

FIGURE 4. Specimens reported from the DX5 outcrop section in Metzger (2005). All specimens in lateral view. 1-3, 5, Pterospathodus eopennatus ssp. n. 2 sensu Männik (1998), Pa element, DLH 4, SUI 132418 (1); Pa element, DLH 6, SUI 132420 (2); Pb element, DLH 6, SUI 132420 (3); and Pa element, DLH 9, SUI 132423 (5). 4, 6-9, Pterospathodus amorphognathoides angulatus, Pa element, DLH 6, SUI 132420 (4); Pb element, DLH 6, SUI 132420 (6); Pa element, DLH 6, SUI 132420 (7); Pb element, DLH 6, SUI 132420 (8); and Pa element, DLH 11, SUI 132425 (9). 10, Aulacognathus bullatus? Pb element, DLH 11, SUI 132425.

identified in Metzger (2005) are re-assigned (see Discussion).

The lithology of the LPC in the GC is widely variable (Figure 5). The base of the LPC is equivalent to the uppermost part of the lower Hopkinton member, and is composed mainly of breccia beds with chert and large limestone interclasts in a slightly sandy mud matrix. The portion of the LPC equivalent to the Farmers Creek Quarry Member contains brecciated chert beds, a bio-dolo-mud- to wackestone with crinoid debris, and bio-mud- to packstones. Chert nodules are scattered throughout. The portion of the LPC equivalent to the Picture Rock Member of the Hopkinton Formation is a green, calcareous shale to mudstone, and the remainder are bio-wacke- to packstones. The por- tion of the LPC equivalent to the BCQ Member of the Scotch Grove Formation is composed of predominantly mudstones with scattered crinoid debris punctuated by occasional bio-wacke- to packstones with crinoid debris and brachiopod fossils. There are some intervals of argillaceous mudstones and calcareous green shales that are more predominant in the middle of the $B C Q$ equivalent in the GC. The BCQ is exposed at the base of the DX5 outcrop and is a bio-dolo-wackestone with occasional chert nodules. The base of the LPC is marked by thinly bedded chert, overlain by a bed of slightly dolomitized crinoidal wackestone with chert nodules (Figure 6). The rest of the outcrop consists of crinoidal wacke- to packstones with occasional intervals containing frequent chert nodules. 


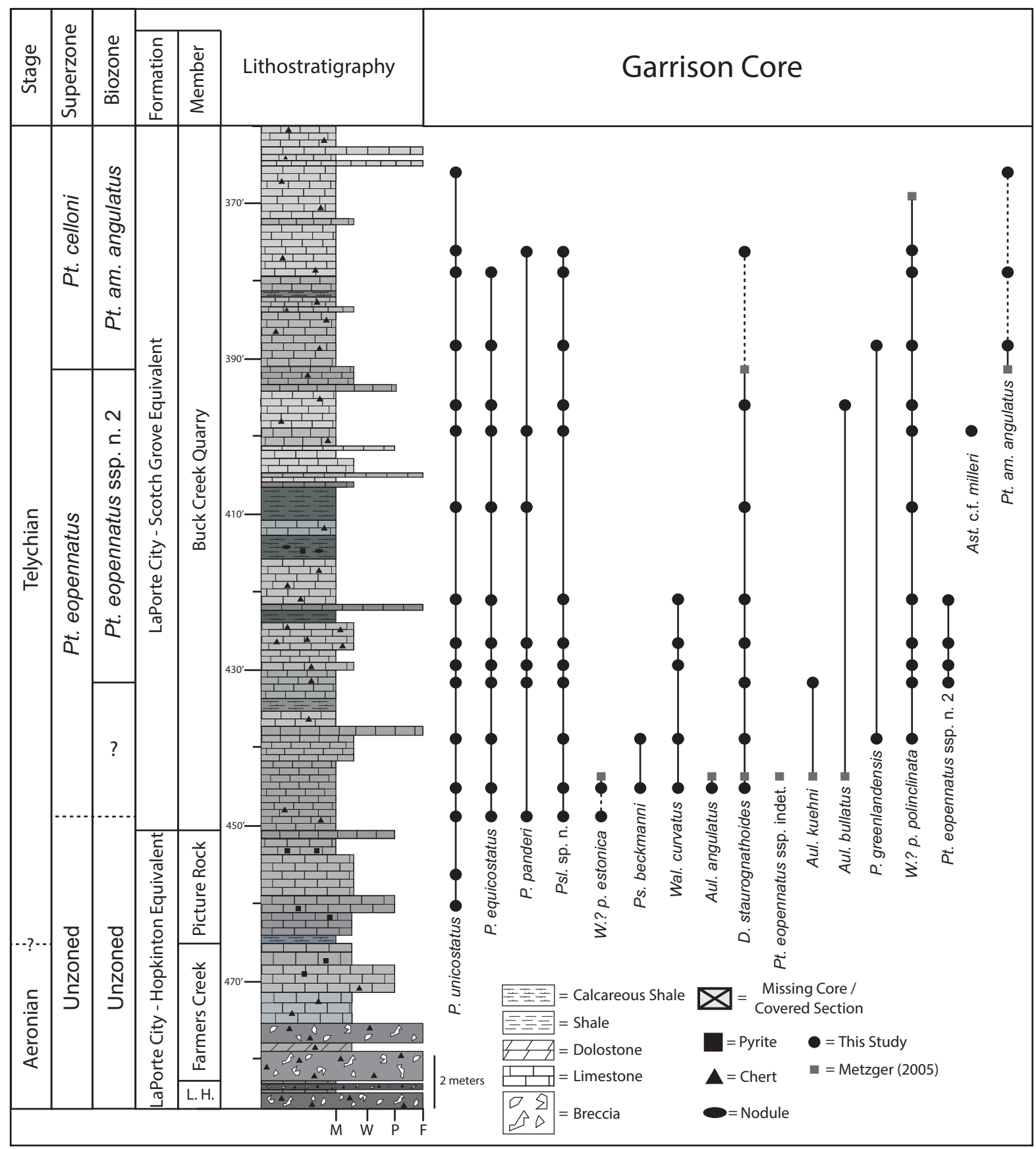

FIGURE 5. Stratigraphic column and conodont range chart for the LPC in the Garrison Core. Dashed lines indicate uncertainty in range. $\mathrm{M}=$ mudstone, $\mathrm{W}=$ Wackestone, $\mathrm{P}=$ Packstone, $\mathrm{F}=$ Floatstone for carbonate lithologies. $\mathrm{L}$. $\mathrm{H}$. $=$ Lower Hopkinton. Log colors represent the color of the rock. Correlation of conodont biozonation to chronostratigraphic units from Cramer et al. (2011).

\section{MATERIAL AND METHODS}

The GC was slabbed, and approximately 0.5 $\mathrm{kg}$ samples were taken at 0.5 to $1.5 \mathrm{~m}(2-4 \mathrm{ft})$ intervals from one-half of the core. Each sample was approximately $25 \mathrm{~cm}$ (10 in) long. Approxi- mately 2.802 to $7.153 \mathrm{~kg}$ samples were collected at 1 to $2 \mathrm{~m}$ intervals from the DX5 outcrop. Samples were then dissolved in $10 \%$ formic acid solution double-buffered with calcium carbonate and calcium phosphate (Jeppsson and Anehus, 1995). 


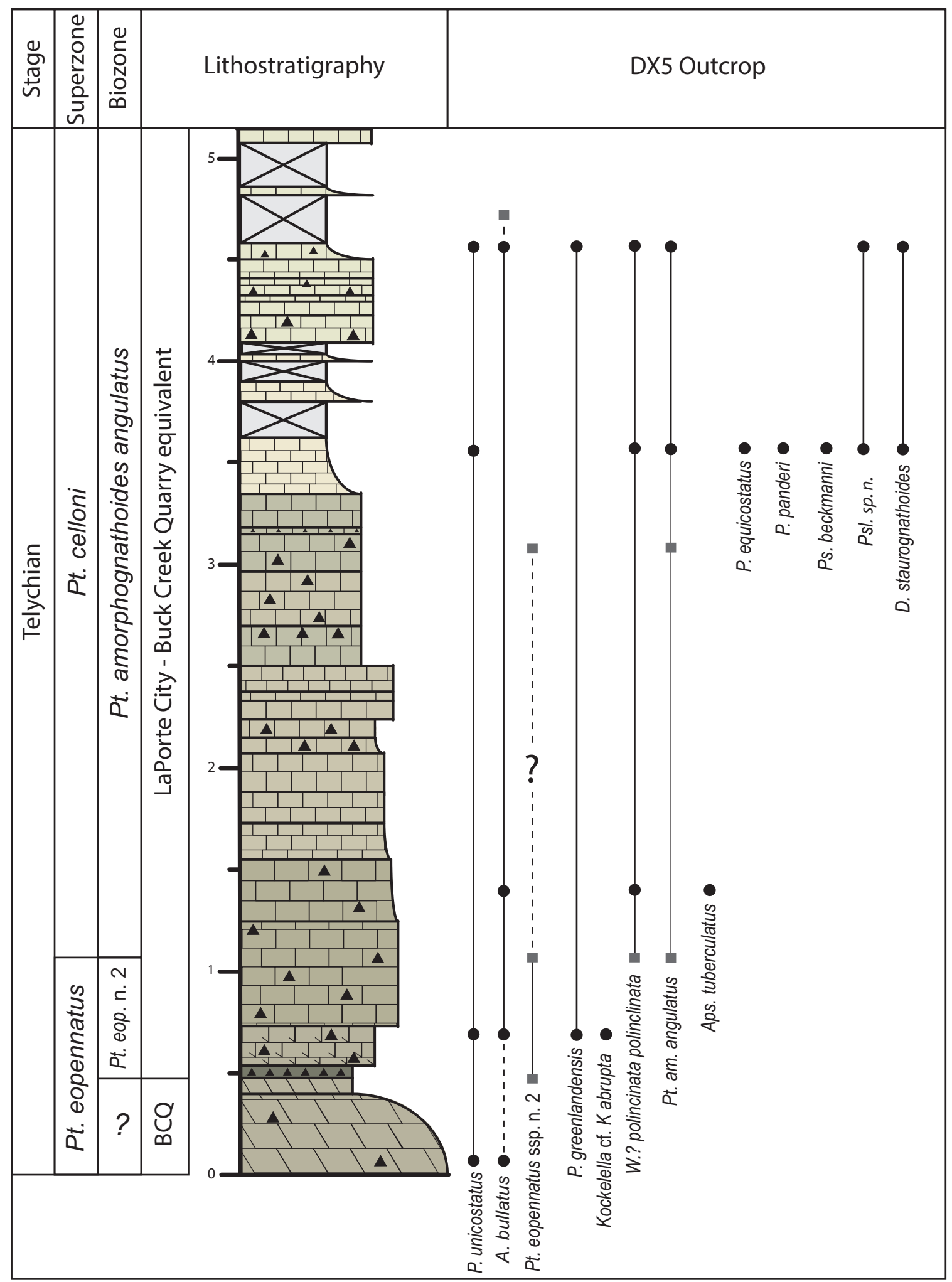

FIGURE 6. Stratigraphic column and conodont range chart for the LPC at the DX5 outcrop. Dashed lines indicate uncertainty in range. See Figure 5 for lithology and symbol legend. The samples included from Metzger (2005) included are DLH 4, DLH 6, DLH 11, and DLH 13, in ascending stratigraphic order. 
The insoluble residue was washed through 1.0 and $0.063 \mathrm{~mm}$ mesh sieves. Density separation was performed on the material from the $0.063 \mathrm{~mm}$ sieve using lithium metatungstate. Samples with relatively high dolomite content were separated at 2.85 $\mathrm{g} / \mathrm{ml}$, and samples with low dolomite content at $2.75 \mathrm{~g} / \mathrm{ml}$. Specimens were picked from the heavy fraction, and photographs were taken of representative specimens using a mounted Canon digital camera and Zerene image stacking software.

All limestone samples from the GC and outcrop readily dissolved in acid after two to three days. Shale samples from the GC were sufficiently calcareous to be disaggregated by acid treatment. The dolostone sample from the base of the DX5 outcrop required a second round of acid treatment to break down remaining dolomite crystals. Conodont yields ranged from eight to 142 elements $/ \mathrm{kg}$ from the GC, with most samples yielding over 50 elements $/ \mathrm{kg}$ (Table 1). Conodont yields were much poorer from samples taken at the DX5 outcrop, with a range of two to 14 elements $/ \mathrm{kg}$ (Table 2). Preservation of the specimens from the GC was generally good to fair, while conodonts from the DX5 outcrop were generally poorly preserved and fragmented. There were rare very-well preserved specimens from both locations. All specimens had a conodont color alteration index (CAl) of 1 , indicating a maximum burial temperature of $80^{\circ} \mathrm{C}$ (Epstein et al., 1977).

Specimens representative of 19 taxa were recovered from the $\mathrm{GC}$, and specimens representative of 13 taxa were recovered from the DX5 outcrop. In total, 21 distinct species and subspecies representing the genera Astrolecignathus, Apsidognathus, Aulacognathus, Distomodus, Kockelella, Oulodus, Panderodus, Pseudolonchodina, Psuedooneotodus, Pterospathodus, Walliserodus, and Wurmiella? were recovered from the LPC (Figures 7-11; Systematic Paleontology section). Samples from both localities were dominated by species with simple-cone apparatuses, with Panderodus unicostatus (Branson and Mehl, 1933) and Panderodus equicostatus (Rhodes, 1953) being the most common.

\section{SYSTEMATIC PALEONTOLOGY}

The element notation of Sweet and Schönlaub (1975), which does not indicate homology of element location and orientation, is primarily used in this study for apparatuses containing ramiform, or ramiform and platform elements. The notation of Purnell et al. (2000) was developed primarily from bedding-plane assemblages of ozarkodinid conodonts, and indicates homologous positions and orientations of each element in the conodont animal. In this paper, it is only applied to members of families Kockelellidae and Spathognathodontidae. For species within these two families, elements previously described as $\mathrm{Sb}$ will be assumed as occupying the $S_{1}$ or $S_{2}$ positions, and be designated as $S_{1 /}$ ${ }_{2}$ elements. Elements previously described as Sc elements will be assumed to have occupied the $S_{3}$ or $S_{4}$ positions, and designated as $S_{3 / 4}$ elements. Synonymies include only illustrated $\mathrm{Pa} / \mathrm{P}_{1}$ elements unless otherwise specified. The locational terms of Sansom et al. (1994) are used for multielement description of the coniform genus Panderodus. Full synonymies are not given for Psuedooneotodus beckmanni, Panderodus unicostatus, P. panderi, and Walliserodus curvatus, but references with specimens recorded from different geographic areas are listed. All specimens are stored in the Paleontology Repository of the Department of Earth and Environmental Sciences at the University of lowa. Figured specimens are each assigned an individual SUI catalogue number, and the SUI numbers of slides containing other specimens from each sample interval are listed in Appendix 1.

Family BALOGNATHIDAE Hass, 1959

Genus APSIDOGNATHUS Walliser, 1964

Type species. Apsidognathus tuberculatus Walliser, 1964.

Diagnosis. Apparatus consists of platform, lenticular, ambalodontan, astrognathodontan, lyriform, compressed, and conical elements. Platform elements are broad, variably ornamented, with several nodose ridges radiating from the center of the element. See Armstrong (1990) and Wang and Aldridge (2010) for thorough diagnosis.

Remarks. The position of the elements within Apsidognathus are unclear. It is likely that the platform element occupied the $\mathrm{Pa}$ position and the lenticular and ambalodontan elements occupied $\mathrm{Pb} /$ Pc positions. Wang and Aldridge (2010) suggested that the ornamented elements (platform, lenticular, ambalodontan, lyriform, compressed) may occupy the $\mathrm{P}$ and $\mathrm{M}$ positions in the apparatus with the $\mathrm{S}$ positions filled by conical and astrognathodontan elements.

\section{Apsidognathus tuberculatus Walliser, 1964} Figure 9.18

*1964 Apsidognathus tuberculatus; Walliser, pl. 5, fig. 1; pl. 12, figs. 16-22; pl. 13, figs. 1-5 (Pa element). 
WAID \& CRAMER: TELYCHIAN CONODONTS FROM IOWA

TABLE 1. Conodont specimens from the Garrison Core.

\begin{tabular}{|c|c|c|c|c|c|c|c|c|c|c|c|c|c|c|c|}
\hline \multicolumn{2}{|l|}{ Sample (m) } & 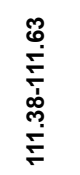 & 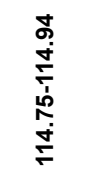 & 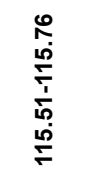 & $\begin{array}{l}\bar{\sigma} \\
\stackrel{\infty}{\infty} \\
\bar{i} \\
\stackrel{\infty}{\infty} \\
\stackrel{\infty}{\rightleftharpoons}\end{array}$ & 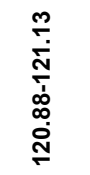 & 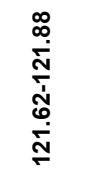 & 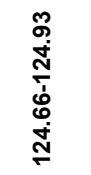 & 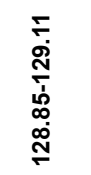 & 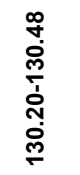 & 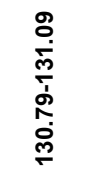 & 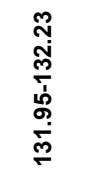 & $\begin{array}{l}\bar{m} \\
\dot{j} \\
\bar{j} \\
\dot{\delta} \\
\dot{j} \\
\dot{m}\end{array}$ & 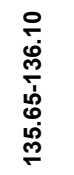 & 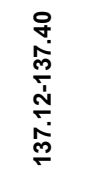 \\
\hline \multirow{2}{*}{\multicolumn{2}{|c|}{$\begin{array}{l}\text { Sample (ft) } \\
\text { Mass (kg) }\end{array}$}} & 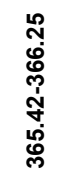 & 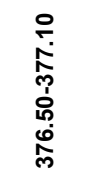 & 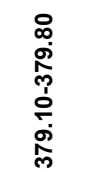 & 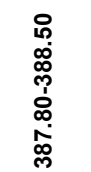 & 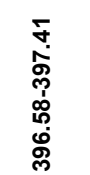 & 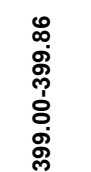 & 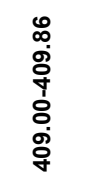 & 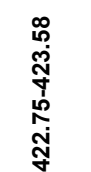 & 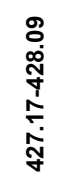 & 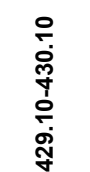 & 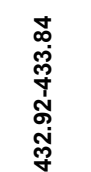 & 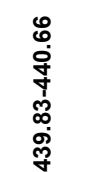 & 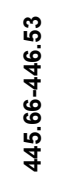 & 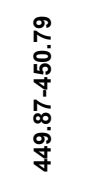 \\
\hline & & 0.52 & 0.523 & 0.526 & 0.522 & 0.544 & 0.555 & 0.562 & 0.554 & 0.52 & 0.521 & 0.551 & 0.561 & 0.56 & 0.561 \\
\hline \multicolumn{2}{|l|}{ Total Conodonts } & 4 & 17 & 31 & 15 & 26 & 33 & 33 & 54 & 74 & 63 & 77 & 42 & 80 & 32 \\
\hline \multirow{3}{*}{$\begin{array}{l}\text { Pterospathodus } \\
\text { amorphognathoides } \\
\text { angulatus }\end{array}$} & $\mathrm{Pb}_{1}$ & & & 1 & & & & & & & & & & & \\
\hline & $\mathrm{Pb}_{2}$ & 1 & & & 1 & & & & & & & & & & \\
\hline & $\mathrm{Sb}_{1}$ & & & 1 & 1 & & & & & & & & & & \\
\hline \multirow{6}{*}{$\begin{array}{l}\text { Pterospathodus } \\
\text { eopennatus ssp. n. } 2\end{array}$} & $\mathrm{~Pa}$ & & & & & & & & 1 & 1 & 1 & 1 & & & \\
\hline & $\mathrm{Pb}_{1}$ & & & & & & 1 & & 3 & 2 & 1 & 1 & & & \\
\hline & $\mathrm{Pc}$ & & & & & & & & & 1 & & & & & \\
\hline & $M$ & & & & & & & & & & 2 & & & & \\
\hline & $\mathrm{Sc}_{1}$ & & & & & & & & 2 & 1 & & & & & \\
\hline & $\mathrm{Sb}_{2}$ & & & & & & & & 1 & 1 & & & & & \\
\hline \multirow{7}{*}{$\begin{array}{l}\text { Aulacognathus sp. } \mathrm{n} . \\
\text { Wurmiella? } \\
\text { polinclinata } \\
\text { polinclinata }\end{array}$} & $\mathrm{P}_{1}$ & & & & & & & & & & 1 & & & 1 & \\
\hline & $\mathrm{P}_{1}$ & & & & & & 1 & & 2 & 3 & 1 & 4 & 3 & & \\
\hline & $\mathrm{P}_{2}$ & & 1 & 1 & 1 & 1 & & 3 & 3 & 2 & 3 & & 2 & & \\
\hline & M & & & & & & & & & & & 1 & & & \\
\hline & $\mathrm{S}_{3 / 4}$ & & & 1 & & & & & & & & & 5 & & \\
\hline & $S_{1 / 2}$ & & & 2 & & 1 & & & 1 & 5 & & 1 & 3 & & \\
\hline & $\mathrm{S}_{0}$ & & 2 & & & & & 3 & & 1 & 1 & & & & \\
\hline \multirow{6}{*}{$\begin{array}{l}\text { Wurmiella? } \\
\text { polinclinata estonica }\end{array}$} & $\mathrm{P}_{1}$ & & & & & & & & & & & & & 3 & \\
\hline & $\mathrm{P}_{2}$ & & & & & & & & & & & & & 3 & \\
\hline & $M$ & & & & & & & & & & & & & 3 & \\
\hline & $\mathrm{S}_{3 / 4}$ & & & & & & & & & & & & & 1 & \\
\hline & $S_{1 / 2}$ & & & & & & & & & & & & & 2 & 1 \\
\hline & $\mathrm{S}_{0}$ & & & & & & & & & & & & & 1 & 2 \\
\hline \multirow{6}{*}{$\begin{array}{l}\text { Psuedolonchodina } \\
\text { sp. n. }\end{array}$} & $\mathrm{Pa}$ & & & 1 & & & & & & & 1 & & & & \\
\hline & $\mathrm{Pb}$ & & & 1 & & 1 & & & 1 & & 3 & & 1 & 1 & 1 \\
\hline & M & & 1 & 1 & & 2 & & & 2 & 2 & & 2 & 1 & & \\
\hline & Sc & & & 4 & 1 & 1 & 2 & & 4 & 4 & 4 & 5 & 2 & & 1 \\
\hline & $\mathrm{Sb}$ & & 1 & 1 & & & & & 1 & & 3 & & 1 & & \\
\hline & $\mathrm{Sa}$ & & & 2 & & 1 & 3 & & 1 & & & & & & \\
\hline \multirow{3}{*}{$\begin{array}{l}\text { Aulacognathus } \\
\text { angulatus }\end{array}$} & $\mathrm{P}_{1}$ & & & & & 1 & & & & 1 & & & 1 & 1 & \\
\hline & P2 & & & & & 1 & & & & & & & & 1 & \\
\hline & $S_{1 / 2}$ & & & & & & & & & & & & & 1 & \\
\hline
\end{tabular}


TABLE 1 (continued).

\begin{tabular}{|c|c|c|c|c|c|c|c|c|c|c|c|c|c|c|c|}
\hline \multicolumn{2}{|l|}{ Sample (m) } & 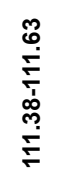 & 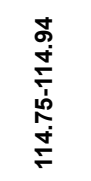 & 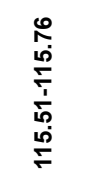 & 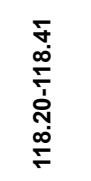 & 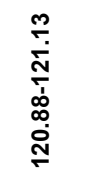 & 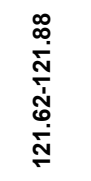 & 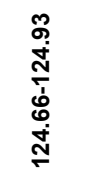 & 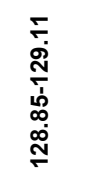 & 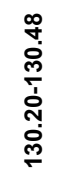 & 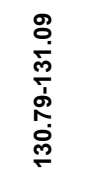 & 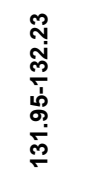 & $\begin{array}{l}\bar{m} \\
\dot{m} \\
\overline{\dot{m}} \\
\dot{\dot{g}} \\
\dot{m}\end{array}$ & 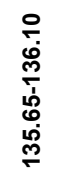 & $\begin{array}{l}\stackrel{P}{\grave{j}} \\
\stackrel{m}{\grave{i}} \\
\stackrel{i}{\dot{m}} \\
\stackrel{m}{m}\end{array}$ \\
\hline \multirow{2}{*}{\multicolumn{2}{|c|}{$\begin{array}{l}\text { Sample (ft) } \\
\text { Mass (kg) }\end{array}$}} & 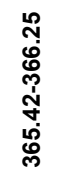 & 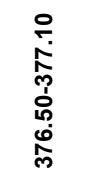 & 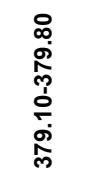 & 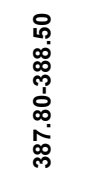 & 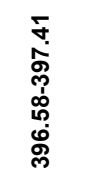 & 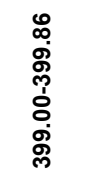 & 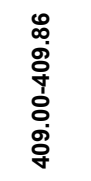 & 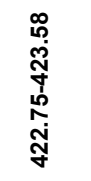 & 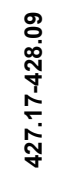 & 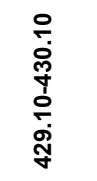 & 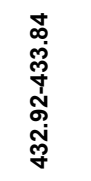 & 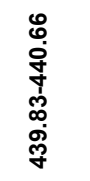 & 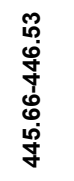 & 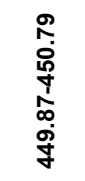 \\
\hline & & 0.52 & 0.523 & 0.526 & 0.522 & 0.544 & 0.555 & 0.562 & 0.554 & 0.52 & 0.521 & 0.551 & 0.561 & 0.56 & 0.561 \\
\hline \multicolumn{2}{|l|}{ Total Conodonts } & 4 & 17 & 31 & 15 & 26 & 33 & 33 & 54 & 74 & 63 & 77 & 42 & 80 & 32 \\
\hline $\begin{array}{l}\text { Aulacognathus } \\
\text { kuehni }\end{array}$ & $\mathrm{P}_{1}$ & & & & & & & & & & & 3 & & & \\
\hline $\begin{array}{l}\text { Aulacognathus } \\
\text { bullatus }\end{array}$ & $\mathrm{P}_{1}$ & & & & & 1 & & & & & & & & & \\
\hline $\begin{array}{l}\text { Astrolecignathus cf. } \\
\text { milleri }\end{array}$ & $\mathrm{Pa}$ & & & & & & 1 & & & & & & & & \\
\hline \multirow{2}{*}{$\begin{array}{l}\text { Distomodus } \\
\text { staurognathoides }\end{array}$} & $\mathrm{Pa}$ & & & & & 1 & & 1 & 1 & 1 & & 2 & & 4 & \\
\hline & $\mathrm{Sb}$ & & 1 & & & & & & 1 & & & & 2 & 3 & \\
\hline \multirow[t]{4}{*}{ Oulodus sp. } & $\mathrm{Pa} ?$ & & & & & & & & & & & & & 1 & \\
\hline & $\mathrm{Pb}$ & & & & 1 & 1 & & & & & & & & & \\
\hline & Sc & & & & & & & & & 1 & & & 2 & 2 & \\
\hline & Sa & & & & & 2 & & & & & 1 & & & & 1 \\
\hline \multirow{3}{*}{$\begin{array}{l}\text { Oulodus cf. petilus } \\
\text { pacificus }\end{array}$} & Sc & & & & & & & & & & & & 1 & & \\
\hline & $\mathrm{Sb}$ & & & & & & & & & 1 & & & & & \\
\hline & $\mathrm{Pb}$ & & & & & & & & & & 1 & & & & \\
\hline \multirow{6}{*}{$\begin{array}{l}\text { Panderodus } \\
\text { unicostatus }\end{array}$} & aeq & & & & & & & & & 1 & & 1 & & & \\
\hline & tort & & 2 & 2 & 1 & 1 & & 1 & & 5 & 1 & 3 & 1 & 2 & 1 \\
\hline & falc & & 1 & 2 & & 1 & & & & 2 & 4 & 4 & & 3 & \\
\hline & grac & & 1 & 2 & 1 & 3 & 3 & 2 & 4 & 18 & 13 & 14 & 1 & 10 & 4 \\
\hline & trunc & & & 1 & 1 & 1 & 1 & & 1 & 4 & 1 & 3 & & 3 & \\
\hline & arc & & 1 & & & & 1 & 1 & & 2 & 2 & 4 & 1 & 1 & 1 \\
\hline \multirow{6}{*}{$\begin{array}{l}\text { Panderodus } \\
\text { equicostatus }\end{array}$} & aeq & & & & & & & & & & & & & 1 & \\
\hline & tort & & & & 1 & & 1 & 2 & & & & 1 & & 1 & \\
\hline & falc & & & & & 1 & 2 & 1 & 2 & 1 & & & & & 1 \\
\hline & grac & & & 2 & 2 & 3 & 6 & 11 & 4 & 3 & 4 & 5 & 1 & 12 & 6 \\
\hline & trunc & & & & & & & $1 ?$ & & & & $1 ?$ & 1 & & \\
\hline & arc & & & & & 1 & 1 & 2 & 1 & 1 & 1 & 2 & & 2 & 1 \\
\hline \multirow[t]{4}{*}{ Panderodus panderi } & falc & & & & & & & & & & 1 & & & & \\
\hline & grac & 1 & & & & & & 3 & 2 & 1 & 6 & 4 & & & 3 \\
\hline & trunc & & 1 & & & & 1 & & 1 & 1 & 2 & 2 & & & \\
\hline & arc & & & & & & & 1 & & & & 1 & & & 1 \\
\hline \multirow{2}{*}{$\begin{array}{l}\text { Panderodus } \\
\text { greenlandensis }\end{array}$} & grac & & & 1 & & & & & & & & & & & \\
\hline & arc & & & & 1 & 1 & & & & & & & 1 & & \\
\hline
\end{tabular}


TABLE 1 (continued).

\begin{tabular}{|c|c|c|c|c|c|c|c|c|c|c|c|c|c|c|c|}
\hline Sample (m) & & 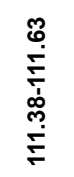 & 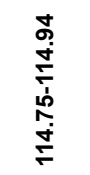 & 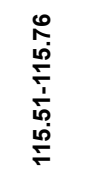 & 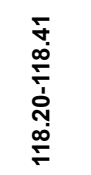 & 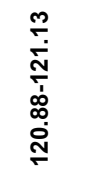 & 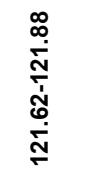 & 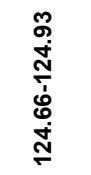 & 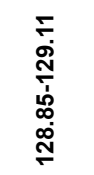 & 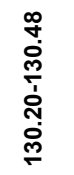 & 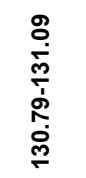 & 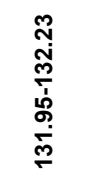 & $\begin{array}{l}\bar{m} \\
\dot{m} \\
\check{\dot{d}} \\
\dot{\delta} \\
\dot{m}\end{array}$ & 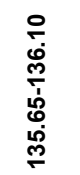 & 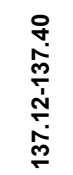 \\
\hline Sample (ft) & & 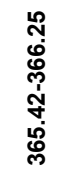 & 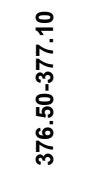 & 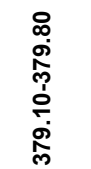 & $\begin{array}{l}0 \\
\stackrel{0}{0} \\
\infty \\
\infty \\
\dddot{m} \\
\vdots \\
\infty \\
\stackrel{\infty}{\infty} \\
\infty\end{array}$ & 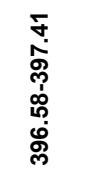 & 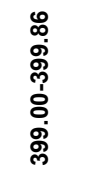 & 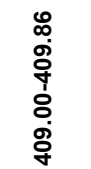 & 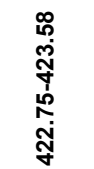 & 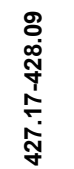 & 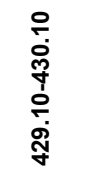 & 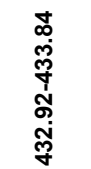 & 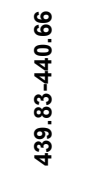 & 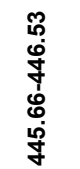 & 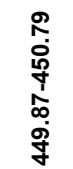 \\
\hline Mass (kg) & & 0.52 & 0.523 & 0.526 & 0.522 & 0.544 & 0.555 & 0.562 & 0.554 & 0.52 & 0.521 & 0.551 & 0.561 & 0.56 & 0.561 \\
\hline Total Conodonts & & 4 & 17 & 31 & 15 & 26 & 33 & 33 & 54 & 74 & 63 & 77 & 42 & 80 & 32 \\
\hline $\begin{array}{l}\text { Panderodus cf. } P . \\
\text { unicostatus }\end{array}$ & grac & & & & & & & & & & & & & & 2 \\
\hline $\begin{array}{l}\text { Panderodus sp. } \\
\text { indet. }\end{array}$ & & 1 & & & & & 6 & 2 & 3 & 3 & & 4 & 4 & 2 & 4 \\
\hline \multirow{4}{*}{$\begin{array}{l}\text { Walliserodus } \\
\text { curvatus }\end{array}$} & dysc & & & & & & & & 2 & & 1 & & 2 & & \\
\hline & curv & & & & & & & & 2 & 1 & 2 & & 1 & & \\
\hline & unicost & & & & & & & & 1 & 1 & 2 & & & & \\
\hline & mlticost & & & & & & & & & 1 & & & & 4 & \\
\hline $\begin{array}{l}\text { Pseudooneotodus } \\
\text { beckmanni }\end{array}$ & & & & & & & & & & & & & 1 & 1 & \\
\hline $\begin{array}{l}\text { Unidentified } \\
\text { ramiforms }\end{array}$ & & 1 & 5 & 5 & 3 & & 3 & & 7 & 2 & & 9 & 4 & 10 & 2 \\
\hline
\end{tabular}

2001 Apsidognathus tuberculatus Walliser; Rickards, Percival, Simpson, and Wright, fig. 2e.

2003 Apsidognathus tuberculatus Walliser; Pyle and Barnes, fig. 18.9-18.10.

2010 Apsidognathus tuberculatus Walliser; Wang and Aldridge, pl. 13, fig. 1 (Pa element; with multielement synonymy through 1999).

Description. Pa elements broad and shield-like in oral view, arched in lateral view. One denticulated blade-like process and one ridge with paired nodes meet in the center of the element at an approximately $160^{\circ}$ angle. Several minor ridges, little more than rows of aligned nodes, extend outwards from the center of the element. Entire platform ornamented by scattered nodes.

Remarks. This orientation of the ridges and style of ornamentation most closely resembles Apsidognathus tuberculatus ssp. n. 3 of Männik (2007a), which is restricted to the Lower Pterospathodus amorphognathoides angulatus Zone.

Material examined. One $\mathrm{Pa}$ element.

Genus ASTROLECIGNATHUS Over and Chatterton, 1987
Type species. Astrolecignathus milleri Over and Chatterton, 1987.

Diagnosis. As reconstructed by Over and Chatterton (1987) apparatus consists of amorphognathiform and pygodiform platform elements that likely occupied $\mathrm{Pa}_{1}$ and $\mathrm{Pa}_{2}(=\mathrm{Pb}$ ?) positions.

Astrolecignathus cf. milleri Over and Chatterton, 1987

Figure 9.17

*1987 Astrolecignathus milleri; Over and Chatterton, 1987, pl. 3, figs. 11-12, 15.

2007a Apsidognathus milleri Over and Chatterton; Männik, fig. 6M.

2008 Apsidognathus milleri Over and Chatterton; Männik, fig. 8L.

Description. Pa elements amorphognathiform with a slightly bowed blade with relatively evenly spaced denticles. Outer process has four aligned nodes that decrease in size away from the blade. Inner process contains three aligned nodes that are less developed than those on the outer process. A flat platform is developed on the outside of the blade and lateral processes. Outer-posterior part of the element is broken. The posterior pro- 
TABLE 2. Conodont specimens from the DX5 outcrop.

\begin{tabular}{|c|c|c|c|c|c|c|}
\hline \multicolumn{2}{|l|}{ Sample (m) } & \multirow{2}{*}{$\begin{array}{c}0.04-0.08 \\
3.078\end{array}$} & \multirow{2}{*}{$\begin{array}{c}0.67-0.74 \\
2.802\end{array}$} & \multirow{2}{*}{$\begin{array}{c}1.34-1.54 \\
6.515\end{array}$} & \multirow{2}{*}{$\begin{array}{c}3.49-3.59 \\
7.153\end{array}$} & \multirow{2}{*}{$\begin{array}{c}4.59-4.61 \\
5.098\end{array}$} \\
\hline Mass (kg) & & & & & & \\
\hline Total Conodonts & & 7 & 15 & 12 & 117 & 24 \\
\hline Pterospathodus sp. Indet. & & & & 1 & & \\
\hline \multirow{4}{*}{$\begin{array}{l}\text { Pterospathodus } \\
\text { amorphognathoides angulatus }\end{array}$} & $\mathrm{Pa}$ & & & & 1 & 1 \\
\hline & $\mathrm{Pb}_{1}$ & & & & 3 & \\
\hline & $\mathrm{Pb}_{2}$ & & & & & 1 \\
\hline & $\mathrm{Sc}_{2}$ & & & & $1 ?$ & \\
\hline \multirow{4}{*}{$\begin{array}{l}\text { Ozarkodina polinclinata } \\
\text { polinclinata }\end{array}$} & $\mathrm{P}_{1}$ & & & & 4 & 3 \\
\hline & $\mathrm{P}_{2}$ & & & & & 1 \\
\hline & M & & & 1 & & 1 \\
\hline & $S_{1 / 2}$ & & & $1 ?$ & & \\
\hline \multirow[t]{5}{*}{ Psuedolonchodina sp. $\mathrm{n}$. } & $\mathrm{Pb}$ & & & & & 1 \\
\hline & M & & & & 1 & \\
\hline & $\mathrm{Sc}$ & & & & 1 & 1 \\
\hline & $\mathrm{Sb}$ & & & & 5 & \\
\hline & $\mathrm{Sa}$ & & & & 1 & \\
\hline \multirow[t]{2}{*}{ Aulacognathus bullatus } & $\mathrm{P}_{1}$ & $1 ?$ & 2 & & & \\
\hline & $\mathrm{P}_{2}$ & & & $1 ?$ & & \\
\hline Apsidognathus tuberculatus & $\mathrm{Pa}$ & & & 1 & & \\
\hline \multirow[t]{2}{*}{ Distomodus staurognathoides } & $\mathrm{Pa}$ & & & & 1 & \\
\hline & $\mathrm{Sb} ?$ & & & & & $1 ?$ \\
\hline Oulodus sp. & $\mathrm{Sc}$ & & & & 2 & \\
\hline Kockelella cf. K. abrupta & $\mathrm{P}_{1}$ & & 1 & & & \\
\hline \multirow[t]{6}{*}{ Panderodus unicostatus } & aeq & & & & 2 & \\
\hline & tort & 1 & & & 4 & 1 \\
\hline & falc & & & & 4 & \\
\hline & grac & 1 & 2 & & 18 & 3 \\
\hline & trunc & & & & 5 & \\
\hline & arc & & & & 3 & 1 \\
\hline \multirow[t]{5}{*}{ Panderodus equicostatus } & tort & & & & 5 & \\
\hline & falc & & & & 3 & \\
\hline & grac & & & & 15 & \\
\hline & trunc & & & & 3 & \\
\hline & arc & & & & 6 & \\
\hline \multirow[t]{3}{*}{ Panderodus panderi } & grac & & & & 3 & \\
\hline & trunc & & & & 4 & \\
\hline & arc & & & & 2 & \\
\hline Panderodus greenlandensis & grac & & 1 & & & 1 \\
\hline Panderodus sp. indet. & & & & & 7 & 8 \\
\hline Pseudooneotodus beckmanni & & & & & 1 & \\
\hline Unidentified ramiforms & & 4 & 9 & 7 & 16 & \\
\hline
\end{tabular}




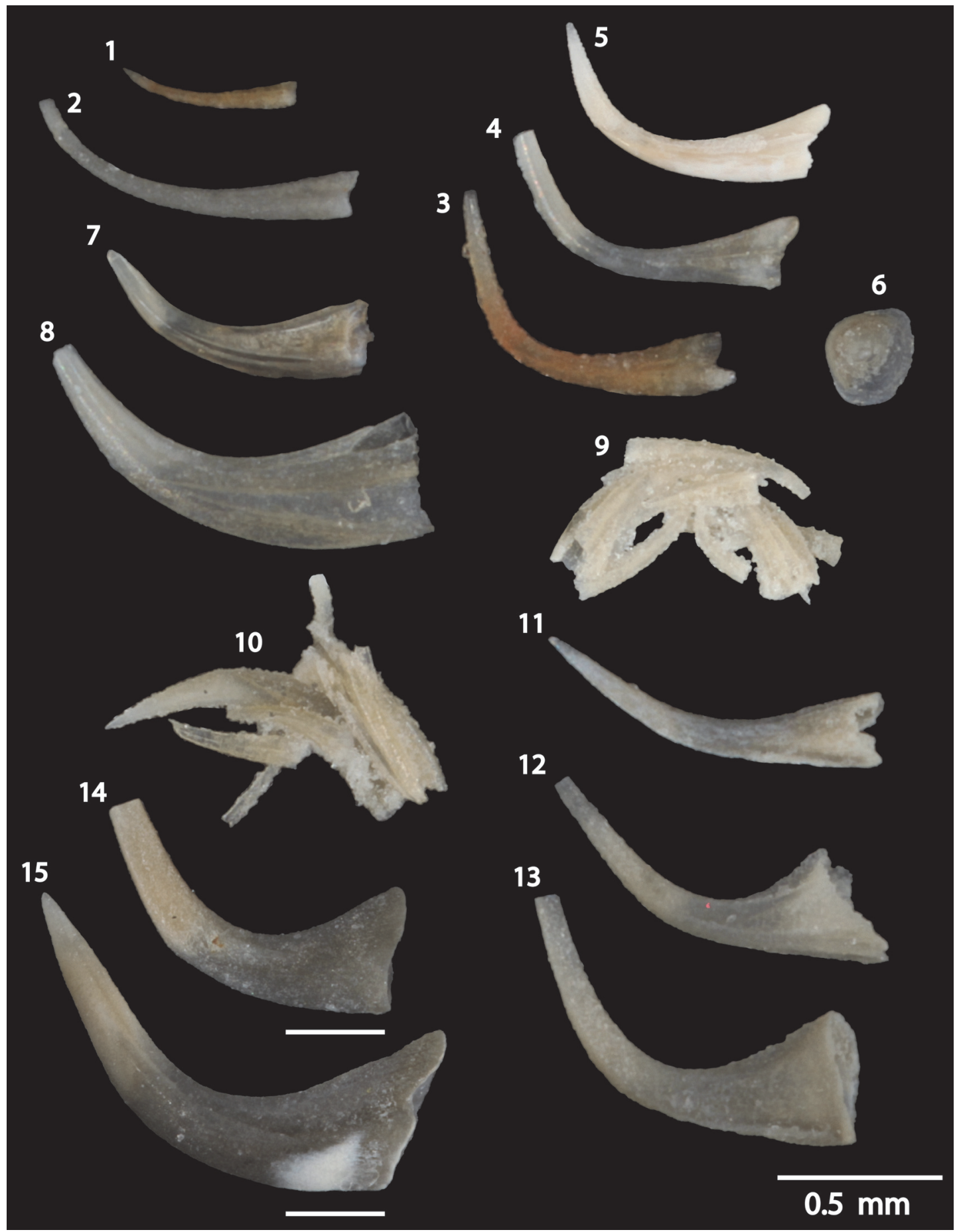

FIGURE 7. Simple cone elements from Garrison Core. All specimens in lateral view except for 6. 1-2, Panderodus equicostatus, graciliform, GC 124.66-124.93 m, SUI 142251 (1) and graciliform, GC 121.92-121.88 m, SUI 142253 (2). 3-5, Panderodus panderi, graciliform, GC 124.66-124.93 m, SUI 142252 (3); arcuatiform, GC 131.95-132.23 m, SUI 142254 (4); and graciliform, GC 111.38-111.63 m, SUI 142255 (5). 6, Psuedooneotodus beckmanni, oral view, GC 135.65-136.10 m, SUI 142256. 7, Panderodus cf. P. unicostatus, falciform, GC 135.65-136.10 m, SUI 142257. 811, Panderodus unicostatus, falciform, GC 131.95-132.23 m, SUI 142258 (8); fused cluster of 8 elements, GC 130.20-130.48 m, specimen destroyed processing for SEM (9); fused cluster of 6 elements, GC 131.95-132.23 m, SUI 142260 (10); and tortiform element, GC 131.95-132.23 m, SUI 142261 (11); 12-13, Walliserodus curvatus, dyscritiform, GC 134.06-134.31 m, SUI 142262 (12) and curvatiform, GC 128.85-129.11 m, SUI 142263 (13). 14-15, Panderodus greenlandensis, arcuatiform GC 118.20-118.41 m, SUI 142264 (14) and graciliform, GC 134.06-134.31 $\mathrm{m}$, SUI 142265 (15). White scale bar represents $0.5 \mathrm{~mm}$. 


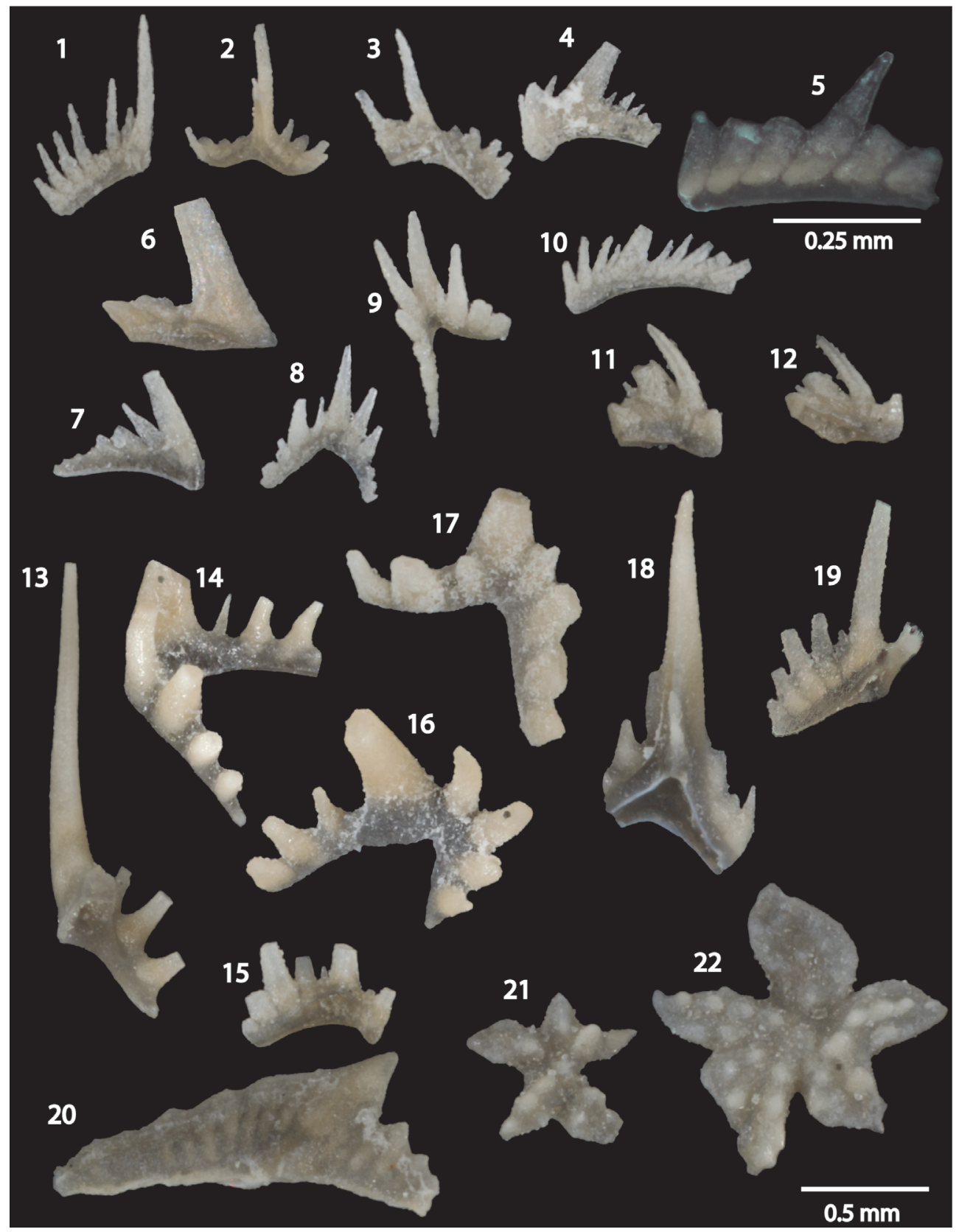

FIGURE 8. Conodonts from the Garrison Core. 1-12, 19, Psuedolonchodina sp. n., Sa element, posterior view, GC 115.51-115.76 m, SUI 152266 (1); Sa element, posterior-aboral view, GC 121.62-121.88 m, SUI 142267 (2); Sb element, anterior view, GC 134.06-134.31 m, SUI 142268 (3); Sc element, lateral view, GC 130.20-130.48, SUI 142269 (4); Sc/M? element fragment, lateral view, GC 128.85-129.11 m, SUI 142270 (5); M element, lateral-posterior view, GC 131.95-132.23 m, SUI 142271 (6); M element, anterior-lateral view, GC 134.06-134.31 m, SUI 142272 (7); Pa element, oral view, GC 134.06-134.31 m, SUI 142273 (8); Pa element, oral view, GC 130.79-131.09m, SUI 142274 (9); Sc element, lateral view, GC 135.65-136.10 m, SUI 142259 (10); Pb element, lateral, and lateral-aboral views, GC 115.51-115.76 m, SUI 142275 (11 and 12); and Pb element, lateral view, GC 130.79-131.09 m, SUI 142276 (19). 13, 15, Oulodus sp. indet., Sa element, anterior-lateral view, GC 137.12-137.40 m, SUI 142277 (13) and Pa element, posterior view, GC 135.65-136.10 m, SUI 142278 (15). 14, 16-17, Oulodus cf. Ou. petilus pacificus, Sc element, lateral view, GC 134.06-134.31 m, SUI 142279 (14); Sb element, posterior-oral view, GC 130.20-130.48 m, SUI 142280 (16); and Pb element, anterior-lateral view, GC 130.79-131.09 m, SUI 142281 (17). 18, 21-22, Distomodus staurognathoides, Sb element, posterior view, GC 134.06-134.31 m, SUI 142282 (18); Pa element, oral view, GC 135.65136.10 m, SUI 142283 (21); and Pa element, oral view, GC 135.65-136.10 m, SUI 142282 (22). 20, Aulacognathus angulatus, $\mathrm{P}_{2}$ element, lateral view, GC 135.65-136.10 m, SUI 142285. 


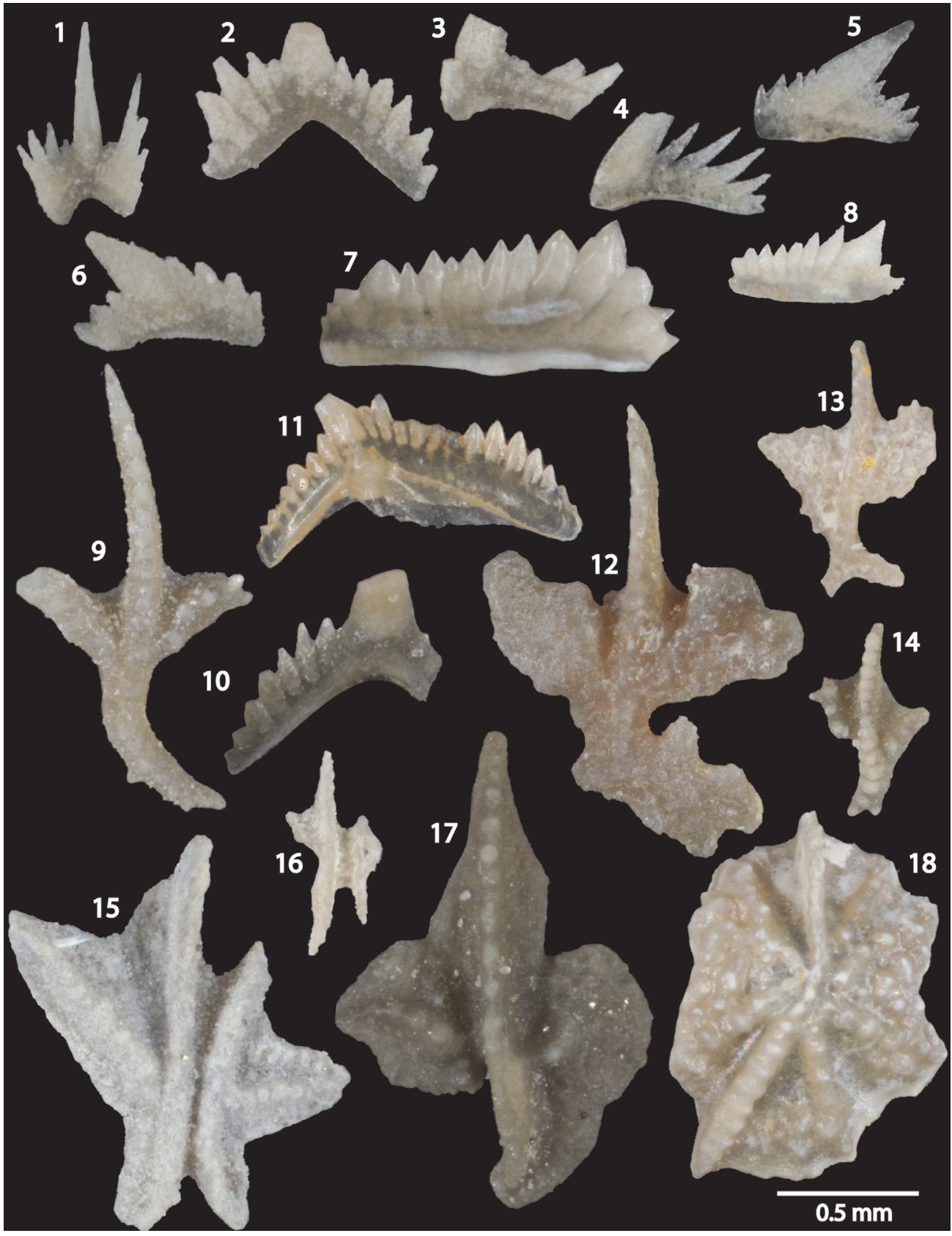

FIGURE 9. Conodonts from the Garrison Core and DX5 outcrop. 1-8, Wurmiella? polinclinata polinclinata, $\mathrm{S}_{0}$ element, anterior view, GC 130.79-131.09 m, SUI 142286 (1); S ${ }_{1 / 2}$ element, posterior view, GC 115.51-115.79 m, SUI 142287 (2); S $3 / 4$ element, lateral view, GC 115.51-115.76 m, SUI 142288 (3); M element, lateral view, GC 130.20130.48 m, SUI 142289 (4); P 2 element, lateral view, GC 130.20-130.48 m, SUI 142290 (5); P 2 element, GC 130.79131.09 m, SUI 142291 (6); $P_{1}$ element, lateral view, DX5 4.59-4.61 m, SUI 142292 (7); and $P_{1}$ element, lateral view, DX5 3.49-3.59 m, SUI 142293 (8). 9-10, Aulacognathus angulatus, $\mathrm{P}_{1}$ element, oral view, GC 135.65-136.10 m, SUI 142294 (9) and $S_{1 / 2}$ ? element, posterior view, GC 135.65-136.10 m, SUI 142295 (10). 11-14, Aulacognathus bullatus, $\mathrm{P}_{2}$ element, lateral view, DX5 1.34-1.54 m, SUI 142296 (11); $\mathrm{P}_{1}$ element, oral view, DX5 4.59-4.61 m, SUI 142297 (12); $P_{1}$ element, oral view, DX5 0.67-0.74 m, SUI 142298 (13); and $P_{1}$ element, oral view, GC 120.88121.13 m, SUI 142299 (14). 15-16, Aulacognathus kuehni, $\mathrm{P}_{1}$ element, oral view, GC 131.95-132.23 m, SUI 142300 (15) and juvenile $P_{1}$ element, oral view, GC 131.95-132.23 m, SUI 142301 (16). 17, Astrolecignathus cf. milleri, Pa element, oral view, GC 121.92-121.88 m, SUI 142302. 18, Apsidognathus tuberculatus, Pa element, oral view, DX5 1.34-1.54 m, SUI 142303. 


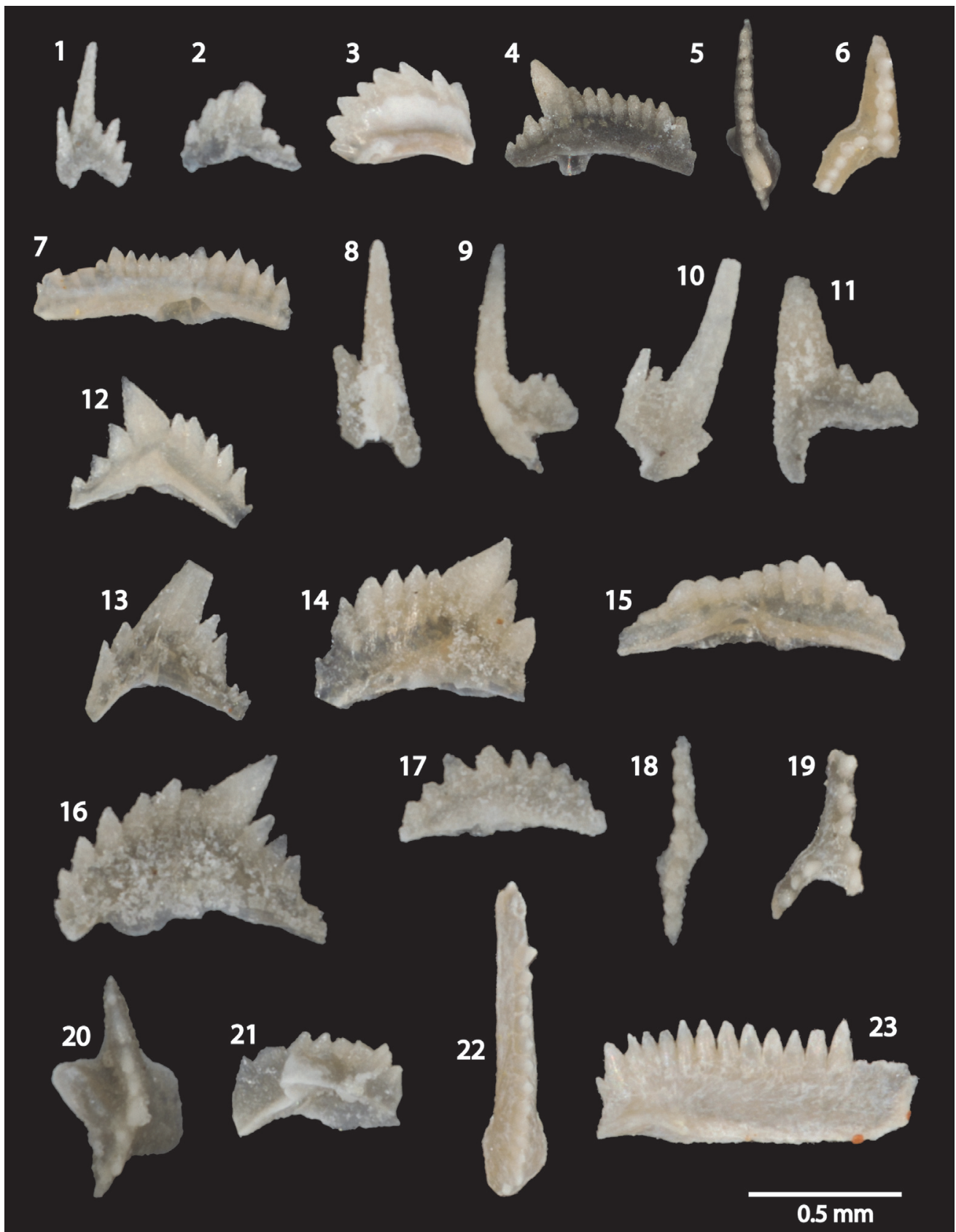

FIGURE 10. Conodonts from the Garrison Core and DX5 outcrop. 1-7, Pterospathodus amorphognathoides angulatus, $\mathrm{Sc}_{1}$ element, posterior view, GC 115.51-115.76 m, SUI 142304 (1); $\mathrm{Pb}_{1}$ ? element, lateral view, GC 115.51115.76 m, SUI 142305 (2); Pb 2 element, GC 111.38-111.63 m, SUI 142306 (3); $\mathrm{Pb}_{1}$ element, inner lateral, and oral views, GC 115.51-115.76 m, SUI 132410 (4 and 5); Pa element, oral view, DX5 3.49-3.59 m, SUI 142307 (6); and Pa element, lateral view, DX5 4.59-4.61 m, SUI 142308 (7). 8-19, Pterospathodus eopennatus ssp. n. 2 sensu Männik (1998), $\mathrm{Sb}_{2}$ element, posterior, and lateral views, GC 130.79-131.09 m, SUI 142309 (8 and 9); Sc 1 element, lat-

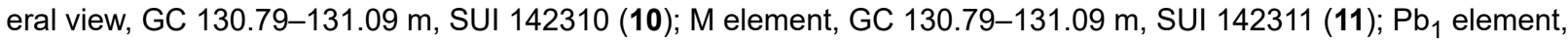
lateral view, GC 128.85-129.11 m, SUI 142312 (12); Pc element, lateral view, GC 130.20-130.48 m, SUI 142313 (13); $\mathrm{Pb}_{1}$ element, lateral view, GC 130.20-130.48 m, SUI 142314 (14); Pa element, lateral view, GC 128.85-129.11 m, SUI 142315 (15); Pb 1 element, lateral view, GC 130.20-130.48 m, SUI 142316 (16); Pa element, lateral, and oral views, GC 130.79-131.09 m, SUI 142317 (17 and 18); and Pa element fragment, oral view, GC 130.20-130.48 m, SUI 142318 (19). 20-21, Aulacognathus sp., juvenile $P_{1}$ element, oral, and lateral-anterior views, GC 135.65-136.10 m, SUI 142319. 22-23, Kockelella cf. K. abrupta, P1 element, oral, and lateral views, DX5 0.67-0.74 m, SUI 142320. 


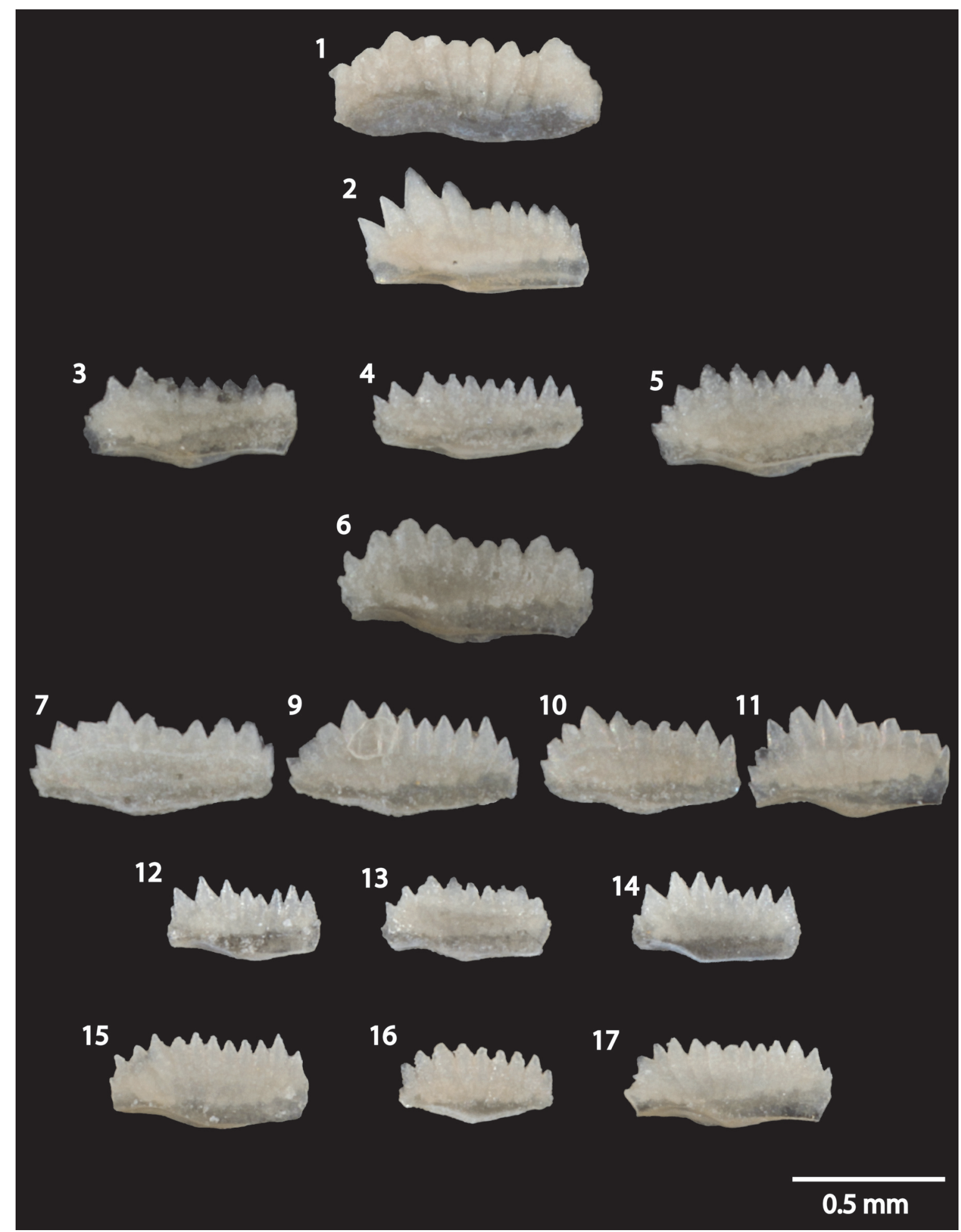

FIGURE 11. $\mathrm{P}_{1}$ elements of Wurmiella? polinclinata estonica and Wurmiella? polinclinata polinclinata. 1-11 are classified as $W$.? polinclinata polinclinata. 12 and 14 show some characteristics of each subspecies, but compare most closely to $W$.? polinclinata polinclinata. 13, 15-17 are classified as W.? polinclinata estonica. All specimens in lateral view. 1, GC 121.92-121.88 m, SUI 142321; 2, GC 128.85-129.11 m, SUI 142322; 3-5, GC 130.20-130.48 m, SUI 142323 (3), 142324 (4), and 142325 (5); 6, GC 130.79-131.09 m, SUI 142326; 7-11, GC 131.95-132.23 m, SUI 142327 (7), 142328 (8), 142329 (9), 142330 (10), and 142331 (11); 12-14, GC 134.06-134.31 m, SUI 142332 (12), 142333 (13), and 142334 (14); and 15-17, GC 135.65-136.10 m, SUI 142335 (15), 142336 (16), and 142337 (17). 
cess is slightly recurved and in oral view is deflected counter-clockwise from the anterior process. The basal cavity is widely expanded, and the basal cavity walls converge to creases under each process.

Remarks. This specimen is tentatively identified as Astrolecignathus milleri because the style of denticulation and nodes, orientation of lateral processes, and the presence of a wide, flat basal platform that extends under the entire element is similar to the holotype (Over and Chatterton, 1987, plate 3 , figure 11). The holotype specimen bears a second, posteriorly oriented process, but that part of the element is broken on our specimen. The antero-lateral process on our specimen is significantly shorter than on the holotype. But the other $\mathrm{Pa}$ element photographed in Over and Chatterton (1987, plate 3 , figure 15 ) bears a smaller process, similar in size to the one on our specimen.

Because the platform lacks any significant ornamentation by nodes, ridges, or webbing, which are a ubiquitous characteristic of species classified as Apsidognathus (e.g., Aps. tuberculatus, Aps. ruginosus Mabillard and Aldridge, 1985), milleri should remain in Astrolecignathus, and should not be assigned to Apsidognathus. Furthermore, the processes on the $\mathrm{Pa}$ elements of species within Apsidognathus tend to radiate from a central point or ridge, whereas the processes of the $\mathrm{Pa}$ elements of Astrolecignathus radiate extend from a point about $1 / 3$ above the most posterior point of the blade. There is also the possibility that the specimens identified as Apsidognathus milleri in Männik (2007a, 2008) are not the same species as the specimens named by Over and Chatterton (1987). The semi-circular arrangement of the denticles on the lateral processes of specimens in Männik $(2007 a, 2008)$ is significantly different from the holotype specimen in Over and Chatterton (1987), where the denticles on the holotype are oriented in completely straight rows.

Material examined. One broken Pa element.

Genus PTEROSPATHODUS Walliser, 1964

Type species. Pterospathodus amorphognathoides Walliser, 1964.

Diagnosis. The apparatus of Pterospathodus as reconstructed by Männik (1998) is composed of 13 paired elements and one un-paired element. Most element morphologies are consistent with $\mathrm{Sa}, \mathrm{Sb}_{1}$, $\mathrm{Sb}_{2}, \mathrm{Sc}_{1}, \mathrm{Sc}_{2}, \mathrm{Sc}_{3}, \mathrm{M}_{1}, \mathrm{M}_{2}, \mathrm{~Pa}, \mathrm{~Pb}_{1}, \mathrm{~Pb}_{2}$, and $\mathrm{Pc}$ designations. Other elements described as carnuliform, carniciform, and "curved element" do not conform to the locational analogies of Sweet and
Schönlaub (1975) when included in the apparatus. $\mathrm{Pa}$ and $\mathrm{Pb}$ elements are primarily used for differentiation of species and subspecies. $\mathrm{Pa}$ elements have a straight to slightly-curved blade, and most species have an inner, anteriorly oriented process, which may bifurcate. Some older species in the genus also have an outer process (e.g., Pt. eopennatus ssp. n. 1 sensu Männik, 1998, morph 5). Younger species have a platform developed under the blade and lateral processes. $\mathrm{Pb}_{1}$ elements are angulate, may be slightly bowed, have a prominent central cusp, and a flared basal cavity. $\mathrm{Pb}_{2}$ elements are angulate or dolabrate, and may have a slightly prominent to minor cusp. Their basal cavities are not as expanded as those in $\mathrm{Pb}_{1}$ elements, but extend along the entire length of the element.

Remarks. If the apparatus reconstruction of Männik (1998) is correct, then the Pterospathodus apparatus contains more than twice as many elements as any conodont apparatus that has been reconstructed based on bedding plane assemblages or fused clusters (e.g., Phragmodus). Elements formerly classified as Carniodus were included in the Pterospathodus apparatus by Männik because each taxa has the same stratigraphic range, and each displays parallel evolutionary patterns (each species of Pterospathodus occurs with a distinct set of Carniodus elements). No elements similar to the carnuliform, carniciform, or curved elements were found in this study, which may be due to the recovery of relatively few Pterospathodus specimens.

Pterospathodus amorphognathoides angulatus (Walliser, 1964)

Figures 4.4, 4.6-4.9, 10.1-10.7

*1964 Spathognathodus pennatus angulatus; Walliser, pl. 14, figs. 19-22 (Pa element).

1998 Pterospathodus amorphognathoides angulatus Walliser; Männik, pl. 2, figs. 1-22, 24-31 (multielement, synonymy through 1998).

2003 Pterospathodus amorphognathoides angulatus Walliser; Loydell, Männik, and Nestor, fig. 14m.

2006 Pterospathodus amorphognathoides angulatus Walliser; Hints, Killing, Männik, and Nestor, pl. 3, fig. 12.

2007 Pterospathodus amorphognathoides angulatus Walliser; Loydell, Kleffner, Mullins, Butcher, Matteson, and Ebert, fig. 6a-d.

2007a Pterospathodus amorphognathoides angulatus Walliser; Männik, fig. 8E-F.

2008 Pterospathodus amorphognathoides angulatus Walliser; Männik, fig. 8O, U.

2010 Pterospathodus amorphognathoides angulatus Walliser; Loydell, Nestor, and Männik, fig. 9r. 
2014 Pterospathodus amorphognathoides angulatus Walliser; Sullivan, Kleffner, and Brett, fig. 7.10.

2015 Pterospathodus amorphognathoides angulatus Walliser; Männik, Loydell, Nestor, and Nõlvak, fig. $8 Q$.

Description. $\mathrm{Sb}_{1}$ element tertiopedate. Posterior process denticulate, straight, and extended down away from the cusp. Outer lateral process with one large denticle, inner lateral process broken. $\mathrm{Pb}_{2}$ element anguliscaphate, with fan-like denticles with fairly uniform height posterior and anterior to a well-developed cusp. Grooves between denticles visible almost down to the base of the element. Basal cavity narrow, extends length of element. $\mathrm{Pb}_{1}$ element anguliscaphate, with well-developed cusp. Denticles extend at similar angles anterior and posterior to the cusp. Denticles anterior to the cusp are much higher than posterior denticles. $\mathrm{Pa}$ element blade-like, with 17 unevenly sized denticles. Denticles widest on anterior and posterior portions of blade. $\mathrm{Pa}$ elements from Metzger (2005) have at least 17 denticles, which is consistent with most morphotypes of the subspecies illustrated in Männik (1998). Denticles situated slightly anterior to the cusp are always much smaller than those posterior to the cusp. Height of blade below where the denticles fuse is similar on anterior and posterior processes.

Remarks. The $\mathrm{Pb}_{1}$ element shown in Figure 10.4-5 was originally reported from the Garrison Core in Metzger (2005), but it was not illustrated. It was identified as a $\mathrm{Pb}_{1}$ element of Pterospathodus amorphognathoides amorphognathoides, but we believe it compares more closely with $\mathrm{Pb}_{1}$ elements of Pterospathodus amorphognathoides angulatus. $\mathrm{Pb}_{1}$ elements of $\mathrm{Pt}$. a. amorphognathoides have a very distinctive narrow platform that is situated just below the denticles on both sides of the anterior and posterior processes, and have a ridge that extends from the basal flare up the cusp. This specimen lacks both of those characteristics, and most closely resembles $P t$. a. angulatus $\mathrm{Pb}_{1}$ elements illustrated in text-figures $7 \mathrm{~F}, 7 \mathrm{P}$, or $8 \mathrm{~T}$ in Männik (1998).

Material examined. One $\mathrm{Sb}_{1}$, three $\mathrm{Sc}_{1}$, one $\mathrm{Sc}_{2}$ ?, four $\mathrm{Pb}_{1}$, three $\mathrm{Pb}_{2}$, one $\mathrm{Pa}$, one $\mathrm{Pa}$ ? elements.

\section{Pterospathodus eopennatus ssp. n. 2 Männik, 1998}

Figures 4.1-4.3, 4.5, 10.8-10.19

*1998 Pterospathodus eopennatus ssp. n. 2; Männik, pl. 1, figs. 1-9, 11-15; pl. 2, figs. 23, 32-41 (multi-element; synonymy for Pt. eopennatus through 1998).

2003 Pterospathodus eopennatus ssp. n. 2 Männik; Loydell, Männik, and Nestor, fig. 14o.

2006 Pterospathodus eopennatus ssp. n. 2 Männik; Hints, Killing, Männik, and Nestor, pl. 3, fig. 9.

2007a Pterospathodus eopennatus ssp. n. 2 Männik; Männik, fig. 6G-H.

Description. $\mathrm{Sb}_{2}$ element tertiopedate. Lateral processes extend from cusp at different angles. Posterior process denticulate. Undenticulated outer lateral process extends down from the bottom of the cusp. Inner lateral process short, with one denticle. $\mathrm{Sc}_{1}$ element bipennate with a short, denticulate anterior process extending down away from the cusp, and a short, denticulate posterior process extending straight away from the cusp. M element dolabrate with an undenticulated anterior process extending straight down from a robust cusp, and a denticulate posterior process extending straight, and at a slight angle down, from the cusp. $\mathrm{Pb}_{1}$ elements angulate to slightly anguliscaphate, denticulate anterior process longer than denticulate posterior process. Cusp and posterior denticles angled posteriorly. Denticles on the anterior process are oriented posteriorly close to the cusp and progressively become more anteriorly oriented farther away from the cusp. Pc element pastinate with a prominent cusp. Anterior process short and angled steeply down from cusp. Posterior process extends straight and intersects cusp at an approximately $90^{\circ}$ angle. Basal cavity flares out beneath cusp into primitive outer lateral process. Pa element in Figure 10.17-18 blade like, with a half-circle profile in lateral view. Denticles are short relative to height of element. Anterior and posterior processes slightly offset in oral view. $\mathrm{Pa}$ element shown in Figure 10.15 relatively long, with 14 denticles on the blade. Denticles highest at the anterior end of the blade, and shortest in the middle of the element. Basal cavity is widest under the shortest denticles. $\mathrm{Pa}$ elements recovered by Metzger (2005) have 16-18 denticles.

Remarks. Two well-preserved $\mathrm{Pa}$ elements were recovered. The one shown in Figure 10.15 most closely resembles morph 1a of Männik (1998), which is characterized by having a long blade with denticles highest on the anterior and posterior portions, and a basal cavity that is widest under the posterior portion of the cusp. The size of the denticles relative to total element height, curvature of the oral surface in lateral view, and geometry of the basal cavity of the specimen shown in Figure 10.16-17 most closely resembles morph $2 b$ of 
Männik (1998). Morph 1 is present in both subspecies of Pterospathodus eopennatus, while morph 2 is only present in Pterospathodus eopennatus ssp. n. 2 sensu Männik (1998). The specimen classified as morph 1 as well as $\mathrm{Pa}$ fragments recovered are assigned to Pterospathodus eopennatus ssp. n. 2 sensu Männik (1998) because they were recovered from sample intervals overlying the morph 2 specimen. The $\mathrm{Pa}$ specimens recovered by Metzger (2005; Figure 4.1-3, 4.5) at the DX5 section most closely resemble morphs $1 \mathrm{a}$ or $1 \mathrm{~b}$ from the Upper Pt. eopennatus ssp. n. 2 Zone (Männik, 1998, text-figure 6).

Material examined. One $\mathrm{Sb}_{1}$, two $\mathrm{Sb}_{2}$, three $\mathrm{Sc}_{1}$, two $\mathrm{M}$, one $\mathrm{Pc}$, seven $\mathrm{Pb}_{1}$, four $\mathrm{Pa}$ elements.

Family BELODELLIDAE Khodalevich and Tschernich, 1973

Genus WALLISERODUS Serpagli, 1967

Type species. Paltodus debolti Rexroad, 1967. Diagnosis. Apparatus contains five simple-cone element morphologies as reconstructed by Zhang and Barnes (2002). Most elements costate and contain a very wide basal cavity that extends to the cusp.

Walliserodus curvatus (Branson and Branson, 1947)

Figure 7.12-13

*1947 Acodus curvatus; Branson and Branson, pl. 82, figs. 9-10, 41, 43.

2002 Walliserodus curvatus Branson and Branson; Won, Blodgett, and Nestor, fig. 7.19-7.21.

2002 Walliserodus curvatus Branson and Branson; Zhang and Barnes, fig. 17.1-17.20, 17.23, 17.24 (multielement; synonymy through 1995).

2003 Walliserodus curvatus Branson and Branson; Pyle and Barnes, fig. 18.2-18.4.

2007a Walliserodus curvatus Branson and Branson; Zhang and Barnes, fig. 8.34-8.38 (multielement).

2007b Walliserodus curvatus Branson and Branson; Zhang and Barnes, fig. 11.1-11.2.

2011 Walliserodus curvatus Branson and Branson; Zhang, fig. 17.1-17.4.

2014 Walliserodus curvatus Branson and Branson; Zhang and Pell, fig. 6.7.

2015 Walliserodus curvatus Branson and Branson; Männik, Loydell, Nestor, and Nõlvak, fig. 8J-K, P.

Description. Elements are coniform with a very large base relative to element size. The walls of basal cavities are thin, except at corners. Curvatiform elements are sharply curved and have weakly developed or no costae. Basal cavity of curvatiform element slightly laterally compressed, giving a pointed oval shape in cross section. Multicostatiform elements have slightly to strongly laterally compressed basal cavities with oval to slightly triangular shaped basal cavities. Several costae extend from corners of the basal cavity to the white matter of the cusp, extending past the point of maximum curvature. Dyscritiform element sharply curved, with a basal cavity that is a symmetrical triangle in cross section. Unicostatiform elements are less sharply curved than the other elements, and have weakly developed costae extending along the outer side of the element.

Material examined. Five dyscritiform, six curvatiform, four unicostatiform, and five multicostatiform elements.

\section{Family DISTOMODONTIDAE Klapper, 1981 Genus DISTOMODUS Branson and Branson, 1947}

Type species. Distomodus kentuckyensis Branson and Branson, 1947.

Diagnosis. Apparatus consists of Sa, Sb, Sc, M, $\mathrm{Pb}$, and $\mathrm{Pa}$ elements. $\mathrm{Pa}$ elements are diagnostic, and consist of a broad platform composed of four to six nodose, to slightly ridged, processes. Basal cavities are highly excavated. $S$ elements of the genus are also distinctive. They can be very robust, have cusps with longitudinal ridges along the sides, and have a very highly excavated basal cavity that is triangular in cross section. See Wang and Aldridge (2010) for thorough diagnosis.

Distomodus staurognathoides (Walliser, 1964)

Figure 8.18, 8.21-22

*1964 Hadrognathus staurognathoides Walliser, pl. 5 fig. 2; pl. 13, figs. 6-15 (Pa elements).

2002 Distomodus staurognathoides Walliser; Zhang and Barnes, fig. 14.1-14.17 (multielement, synonymy through 1999).

2003 Distomodus staurognathoides Walliser; Pyle and Barnes, fig. 16.16.

2006 Distomodus staurognathoides Walliser; Hints, Killing, Männik, and Nestor, pl. 3, fig. 15.

2007 Distomodus staurognathoides Walliser; Loydell, Kleffner, Mullins, Butcher, Matteson, and Ebert, fig. $6 \mathrm{bb}$.

2007a Distomodus staurognathoides Walliser; Zhang and Barnes, fig. 9.26.

2013 Distomodus staurognathoides Walliser; Männik, Miller, and Hairapetian, fig. 4e.

2014 Distomodus staurognathoides Walliser; Sullivan, Kleffner, and Brett, figs. 7.13-7.14, 8.4.

2015 Distomodus staurognathoides Walliser; Loydell, Frỳda, and Gutiérrez-Marco, fig. 8b. 
Description. Pa element stellate and broad, with five processes of varying width and curvature originating from the middle of the element. All processes nodose. Nodes are aligned in fairly even rows on small specimens. On large specimens, some nodes are aligned, and others are scattered over each process. Sb elements are tertiopedate. Lateral processes denticulate, and extend laterally and slightly posteriorly from the cusp at differing angles. Posterior process short with two denticles.

Material examined. Eight Sb, elevenPa elements.

Family KOCKELELLIDAE Klapper, 1981

Genus AULACOGNATHUS Mostler, 1967

Type species. Aulacognathus kuehni Mostler, 1967.

Diagnosis. Apparatus composed of $S_{0}, S_{1 / 2}, S_{3 / 4}$, $M, P_{1}$, and $P_{2}$, elements. $P_{1}$ element has a ridged or nodose main blade with ridged, nodose, and/or lobate lateral processes. Some species have a platform developed between the lateral ridges and blade that may contain nodes. $\mathrm{P}_{2}$ element anguliscaphate with a small outer lateral process extending from the cusp.

\section{Aulacognathus angulatus Bischoff, 1986}

Figures 8.20, 9.9-9.10

*1986 Aulacognathus angulatus; Bischoff, pl. 3, figs. 15-24 ( $P_{1}$ and $P_{2}$ elements)

Description. $\mathrm{P}_{1}$ element consists of a blade with two anteriorly oriented lateral processes that intersect the blade at nearly the same point. Blade denticulate. Anterior portion of the blade bowed. Posterior portion curved and slightly deflected outwardly. Lateral processes narrow, with aligned nodes. A small, very steep platform is developed between the lateral processes and the blade. $\mathrm{P}_{2}$ element angulate. Posterior process deflected inwardly. White matter distributed almost to the base of the element on both the anterior and posterior processes. $S_{1 / 2}$ ? element alate, with arched, excavated, and thickly-walled basal cavity. Denticles are widely separated and oval shaped in cross section. White matter extends below denticles in near-rectangles and is separated by translucent material.

Remarks. This rare species has been previously recovered only from an unnamed limestone unit in New South Wales, Australia (Bischoff, 1986, p. 9, locality number 9). Bischoff assigned the unit to the Stimulograptus sedgwickii or Spirograptus turriculatus graptolite biozones, which correlate to the upper Aeronian to lower Telychian (Cramer et al., 2011). Our specimens were recorded with cono- donts from the lower Telychian Pt. eopennatus Superzone.

Material examined. One $S_{1 / 2}$, one $P_{2}$, one $P_{1}$ element.

Aulacognathus bullatus Nicoll and Rexroad, 1968 Figure 9.11-14

*1968 Neospathognathodus bullatus Nicoll and Rexroad, pl. 1, figs. 5-7 (Pa element).

1990 Aulacognathus bullatus Nicoll and Rexroad; Armstrong, pl. 6 figs. 1-2, 4-7 (multielement; synonymy through 1987).

1991 Aulacognathus bullatus Nicoll and Rexroad; McCracken, pl. 3, fig. 1.

2007a Aulacognathus bullatus Nicoll and Rexroad; Männik, fig. 8T.

2007b Aulacognathus bullatus Nicoll and Rexroad; Zhang and Barnes, fig. 13.18.

2015 Aulacognathus bullatus Nicoll and Rexroad; Bancroft, Brunton, and Kleffner, fig. $5 \mathrm{H}$.

Description. $\mathrm{P}_{1}$ element consists of a denticulate, blade-like anterior process that continues as nodose or ridged carina on the platform part of the element. Posterior process bifurcated, with longer process deflected strongly inwardly. Two lobate lateral processes extend anteriorly from the middle of the element. The lateral processes are ornamented by ridges and nodes. Ridges more pronounced on larger specimens. $P_{2}$ element angulate with a small inner lateral process extending from below the cusp. Cusp is reclined posteriorly. The anterior process is longer than the posterior process and higher in lateral view. Denticles smaller on the posterior process than on the anterior and progressively diminish in size away from the cusp. White matter extends to base of denticles on the anterior process and almost to the base of the element on the posterior process.

Remarks. The $\mathrm{P}_{1}$ specimen from the Garrison Core (Figure 9.14) has significantly fewer ridges or nodes on the processes than the specimens from the DX5 outcrop. But the slight development of ridges on the bifurcated posterior process, development of paired and uneven nodes on the outer lateral process, and the connection of the nodes on the inner lateral process to the carina justify its assignment to this species. The specimen from the Garrison Core is much smaller than those from the DX5 outcrop, so it is interpreted as being a juvenile. The $\mathrm{P}_{2}$ specimen from the DX5 outcrop is tentatively assigned to Aulacognathus bullatus because no diagnostic $P_{1}$ elements were found in the same sample.

Material examined. One $P_{2}$ ?, four $P_{1}$ elements. 
Aulacognathus kuehni Mostler, 1967

Figure 9.15-16

*1967 Aulacognathus kuehni; Mostler, pl. 1 figs. 21, 24-25 (Pa element)

1972 Aulacognathus kuehni Mostler; Aldridge, pl. 2 fig. 6.

1974 Aulacognathus kuehni Mostler; Klapper and Murphy, pl. 1, figs. 2-3.

1975 Aulacognathus kuehni Mostler; Aldridge, pl. 3, fig 13.

1983 Aulacognathus kuehni Mostler; Nowlan, fig. 4I.

1986 Aulacognathus kuehni Mostler; Bischoff, pl. 5, figs. 10-16 ( $\mathrm{Pa}$ and $\mathrm{Pb}$ elements).

1987 Aulacognathus kuehni Mostler; Over and Chatterton, pl. 3 fig. 20.

2007a Aulacognathus kuehni Mostler; Männik, fig. 6B.

2008 Aulacognathus kuehni Mostler; Männik, fig. 8J.

2014 Aulacognathus kuehni Mostler; Sullivan, Kleffner, and Brett, fig. 7.1.

Description. $P_{1}$ element broad, stellate, with a straight primary carina that continues as a blade at the anterior end of the element. A nodose outer lateral process extends from the point where the main carina is replaced by the blade. A secondary, bowed carina runs roughly parallel to the main carina. The primary and secondary carinas are separated by a deep groove. Secondary carina is replaced by a low blade in the anterior portion of the element. A nodose and wide inner lateral process extends from the middle of the secondary carina. Secondary carina less bowed, and lateral processes less developed on juvenile specimen.

Material examined. Three $\mathrm{P}_{1}$, one $\mathrm{P}_{1}$ fragment.

\section{Aulacognathus sp.}

Figure 10.20-21

Description. $\mathrm{P}_{1}$ element slightly stellate, blade slightly bowed in oral view, with an outer lateral lobe and an anteriorly-oriented inner lateral process. Denticles in center of blade slightly taller than those towards the posterior or anterior ends of the element. Inner lateral process contains small denticles. Outer lateral lobe without ornamentation. Basal cavity wide and highly excavated under basal platform.

Remarks. The geometry of the blade and inner lateral process and highly excavated basal cavity is similar to many species of Aulacognathus. However, no features diagnostic of a species within Aulacognathus are present. The specimens are very small and are interpreted as being juveniles, so either the specimens may represent a heretofore undescribed species, or diagnostic features are not developed.
Material examined. Two $\mathrm{P}_{1}$ elements.

Genus KOCKELELLA Walliser, 1957

Type species. Kockelella variablis Walliser, 1957. Diagnosis. Apparatus consists of $S_{0}, S_{1 / 2}, S_{3 / 4}, M$, $P_{1}$, and $P_{2}$ elements. $P_{1}$ element has a straight, blade-like anterior and posterior processes that may be straight, or at an angle, to one another. The posterior process is often shorter than the anterior, and a basal cavity flares out to a platform where the two processes meet. Basal cavities are excavated, and platforms vary in size, depending on the number and size of lateral processes. $S$ and $M$ elements contain widely spaced, laterally compressed denticles.

\section{Kockelella cf. K. abrupta (Aldridge, 1972) sensu Aldridge (1972) \\ Figure 10.22-23}

1972 Spathognathodus cf. S. abruptus; Aldridge, 1972, pl. 4, fig. 8 (Pa element).

2005 Kockelella cf. K. abruptus (Aldridge); Metzger, pl. 1, figs. 18-20.

Description. $\mathrm{P}_{1}$ element blade-like, very slightly bowed in oral view. Lower profile of element straight in lateral view except around basal cavity. Denticles closely spaced, not fused, and fairly uniform in size along length of element. Anterior process makes up the majority of the blade, and the posterior process consists of four denticles at a slightly different orientation than those on the anterior process. Basal cavity is very small and flares outwardly into a small, asymmetric basal platform where the outer side is larger than the inner. The posterior-most denticles are distinctively shorter than the rest of the denticles.

Remarks. Aldridge (1972) recorded a single Fronian age (= upper Aeronian to lower Telycian) Spathognathodus specimen from the Llandovery type area in Wales, with a morphology similar to $\mathrm{P}_{1}$ elements of both Kockelella ranuliformis (Walliser, 1964), and Kockelella abruptus (Aldridge, 1972). Metzger (2005) and this study recovered three specimens from the DX5 outcrop area nearly identical to the one from Aldridge (1972, plate 4, figure 8 ), together with conodonts diagnostic of the Pterospathodus eopennatus ssp. n. 2 and Pt. amorphognathoides angulatus zones (lower to middle Telychian).

Aldridge (1972) considered Spathognathodus cf. abruptus to be a transitional form between $S$. abruptus and S. ranuliformis Walliser (1964). The occurrence of this morphology in the same interval in the East-Central lowa Basin provides further evidence for this hypothesis. Too few specimens 
exhibiting this morphology have been found to officially assign it a species or subspecies classification with adequate description, but this distinctive morphology may provide biostratigraphic utility.

Material examined. One $\mathrm{P}_{1}$ element.

Family PANDERODONTIDAE Lindström, 1970 Genus PANDERODUS Ethington, 1959

Type species. Paltodus unicostatus Branson and Mehl, 1933.

Diagnosis. Apparatus consists of one unpaired (aequaliform) and five paired (tortiform, falciform, graciliform, truncatiform, arcuatiform) coniform element morphologies. The graciliform elements can be further subdivided in most species. Deep furrows extending most of the length of the elements, expanded basal cavities, and round to slightly laterally compressed basal cavities are characteristic of Panderodus. In most reconstructions of panderodontid apparatuses, different element types can be distinguished by the degree of curvature and asymmetry of the elements, the amount of torsion of the cusps, lateral compression of elements, and ornamentation by costae. See Sansom et al. (1994) for thorough description of the Panderodus apparatus, and Sansom and Smith (2005) for diagnosis of genus.

Panderodus equicostatus (Rhodes, 1953)

Figure 7.1-2

*1953 Paltodus equicostatus; Rhodes, pl. 21, figs. 106-109.

2005 Panderodus equicostatus Rhodes; Sansom and Smith, pl. 1, figs. 26-28 (synonymy through 1994).

2008 Panderodus equicostatus Rhodes; Calner, Eriksson, Clarkson, and Jeppsson, fig. 4A.

2008 Panderodus equicostatus Rhodes; Männik, fig. 10X-Z, BB, CC.

2015 Panderodus equicostatus Rhodes; Jarochowska and Munnecke, fig. 7M, N; fig. 8I-L.

Description. Elements are long, slender, and fairly straight for $2 / 3$ to $3 / 4$ of the length of the element. White matter restricted to the tips of the cusps, where the most curvature occurs. Cusps are very short on most specimens, with the exception of truncatiform elements, where the cusp about $1 / 4$ of the total length of the element. Furrows are very narrow, and extend most of the length of the element. See Sansom and Smith (2005) for detailed species diagnosis, and Sansom et al. (1994) for illustration of apparatus (figured as Panderodus acostatus).

Remarks. This species can be differentiated from Panderodus unicostatus by the distribution of white matter and the overall curvature of the elements. The white matter in $P$. equicostatus elements is restricted to the tips, and the anterior margin of the element is less rounded in lateral view than most specimens of $P$. unicostatus.

Material examined. One aequaliform, eleven tortiform, eleven falciform, seventry-four graciliform, six truncatiform, and eighteen arcuatiform elements.

Panderodus greenlandensis Armstrong, 1990

Figure 7.14-15

*1990 Panderodus greenlandensis; Armstrong, fig. 33, figs. 1-8 of pl.15.

2007 Panderodus greenlandensis Armstrong; Loydell, Kleffner, Mullins, Butcher, Matteson, and Ebert, fig. 6W.

2009 Panderodus greenlandensis Armstrong; Munnecke and Männik, fig. 5T-W.

2009 Panderodus greenlandensis Armstrong; Szaniawski, fig. 1B.

Description. Elements have a very broad basal cavity that tapers sharply towards the cusp. Striations are prominent on the walls of the basal cavity, and do not extend along length of entire element. $A$ deep furrow runs most of the length of the element tapering from wide at the base to narrow farther up the cusp. Both the arcuatiform and the graciliform specimens are very robust and sharply curved. The aboral margin of the arcuatiform element is fairly flat until the point of maximum curvature. It has a pronounced, rounded costa on the furrowed face of the element. The cusp twists slightly towards the unornamented side of the element. The aboral margin of the graciliform element is smoothly curved. The white matter in the graciliform element is restricted to the middle of the cusp, does not extend to the tip, and extends down to the point of maximum curvature. See Armstrong (1990) for thorough description.

Remarks. Panderodus greenlandenis can be differentiated from large specimens of $P$. unicostatus by the shape of the basal cavity, which is larger, and tapers more strongly towards the cusp than that of $P$. unicostatus. Both species have striations, but those on $P$. greenlandensis are more pronounced and are restricted to the basal cavity region. The striations on $P$. unicostatus extend most of the way up the element.

Material examined. Two graciliform and four arcuatiform elements.

Panderodus panderi (Stauffer, 1940)

Figure 7.3-5

*1940 Paltodus panderi Stauffer, pl. 60, figs. 8, 9. 
1975 Panderodus panderi Stauffer; Sweet Sweet, Thompson, Satterfield, pl. 1, fig. 12 (synonymy through 1975).

1977 Panderodus panderi Stauffer; Barnes, pl. 3, fig. 21.

1981 Panderodus panderi Stauffer; Nowlan and Barnes, pl. 6, figs. 3-4, 14.

1994 Panderodus panderi Stauffer; Bauer, fig. 4.314.32.

1995 Panderodus panderi Stauffer; Zhen and Webby, pl. 3, figs. 23-25.

2002 Panderodus recurvatus Rhodes (1953); Zhang and Barnes, fig. 16.1-16.27 (synonymy of $P$. recurvatus through 1995).

2004 Panderodus recurvatus Rhodes; Farrell, pl. 3, figs. 9, 12-13.

2007b Panderodus recurvatus Rhodes; Zhang and Barnes, fig. 10.27-10.23.

2009 Panderodus recurvatus Rhodes; Corriga and Corradini, fig. 6P.

2010 Panderodus panderi Stauffer; Wang and Aldridge, pl. 5, figs. 1-17.

2012 Panderodus recurvatus Rhodes; Corradini and Corriga, fig. 6A.

2015 Panderodus panderi Stauffer; Jarochowska and Munnecke; fig. 8W.

Description. The point of maximum curvature in falciform, tortiform, and arcuatiform elements occurs approximately at the halfway point of the length of the specimens. The point of maximum curvature on graciliform elements is roughly $2 / 3$ to $3 / 4$ of the distance from the base to the cusp. The posterior margin of arcuatiform elements relatively straight before and after the point of maximum curvature. The posterior margins of the other element types curve continuously along the length of the elements. In all element types, white matter extends from the tip of the cusp down to, or near, the point of maximum curvature. Fine striations extend from the base most of the way to the white matter. Furrows terminate at a conspicuous notch at the base of the specimens. The elements are moderately laterally compressed. See Zhang and Barnes (2002) and Jeppsson (1997) for thorough descriptions and species diagnoses.

Remarks. We follow the suggestion of Jeppsson (1997) that $P$. recurvatus is a junior synonym of $P$. panderi. Zhang and Barnes (2002) suggested that $P$. recurvatus may not be a member of genus Panderodus, if their inclusion of double-furrowed elements that lack lateral costae in the apparatus is valid. No specimens were recovered fitting this description.

The distribution of white matter and the lateral extent of striations can be used to distinguish $P$. panderi from small specimens of $P$. greenlandensis. The white matter extends all the way to the tip of the cusp in $P$. panderi, and the striations are not restricted to the walls of the basal cavity, as they are for $P$. greenlandensis. Striations on the base are much more pronounced on $P$. greenlandensis.

Material examined. One falciform, twenty-three graciliform, twelve truncatiform, and five arcuatiform elements.
Panderodus cf. P. unicostatus (Branson and Mehl, 1933)
Figure 7.7

*1933 Paltodus unicostatus; Branson and Mehl, pl. 3 fig. 3

Description. Elements are gently curved. White matter occupies approximately $1 / 4$ of the length of the elements. Fine striations extend across the entire length of the elements. The anterior margin of the element is pinched into a small keel that extends as a ridge from the base to the midpoint of the element. Edges of furrow are slightly raised into costae. A single costa ornaments the unfurrowed face.

Remarks. The distribution of white matter, extent of striations, and general shape of the elements are similar to graciliform elements of Panderodus unicostatus. However, the position of costae and keel along the anterior margin are not characteristic of graciliform elements of that species.

Material examined. Two graciliform elements.

Panderodus unicostatus (Branson and Mehl, 1933) Figure 7.8-11

*1933a Paltodus unicostatus; Branson and Mehl, pl. 3 fig. 3.

1997 Panderodus unicostatus Branson and Mehl; Smelror, Cocks, Mørk, Neuman, and Nakrem, fig. 11D.

2002 Panderodus unicostatus Branson and Mehl; Zhang and Barnes, fig. 15.1-15.24 (synonymy through 1995).

2004 Panderodus unicostatus Branson and Mehl; Farrell, pl. 4, figs. 10-13.

2007b Panderodus unicostatus Branson and Mehl; Zhang and Barnes, fig. 10.16-10.22.

2009 Panderodus unicostatus Branson and Mehl; Corriga and Corradini, fig. 60.

2009 Panderodus unicostatus Branson and Mehl; Munnecke and Männik, fig. 5X-Z.

2010 Panderodus unicostatus Branson and Mehl; Wang and Aldridge, pl. 6, figs. 1-18.

2015 Panderodus unicostatus Branson and Mehl; Jarochowska and Munnecke, fig. 7L, 8G-H. 
2016 Panderodus unicostatus Branson and Mehl; Waid and Over, fig. 3.28.

Description. Posterior margins of all element types except for arcuatiforms are gently curved along entire length. The posterior margin of arcuatiform elements are generally straight. Falciform elements are markedly more robust than other element types and have slightly laterally compressed basal cavities. Tortiform elements orally compressed with the cusp twisting away from the face with a subtle furrow. Aequaliform elements are small yet robust and symmetrical with furrows on each face. Graciliform elements are more slender than other element types and exhibit a varying degree of curvature. Truncatiform elements are similar to graciliform elements, but smaller, and more curved. White matter extends $1 / 4$ to $1 / 3$ of the length of the element from the tip in all element types. Furrows are wide at the base and narrow towards the tip of the element. Fine striations are visible along the entire length of specimens. See Cooper (1975), Barrick (1977), and Sansom et al. (1994) for thorough description and apparatus reconstruction.

Remarks. Panderodus unicostatus was the dominant coniform species throughout the majority of the LaPorte City Formation. Several fused clusters of $P$. unicostatus were recovered. Most of the elements within the clusters appear to be currentaligned (e.g., Figure 7.10), indicating that they are not in the orientation that they were in the conodont animal. Two clusters were recovered that are not imbricated (e.g., Figure 7.9), indicating that the elements may be in orientations similar to their orientations in the organism.

Material examined. Two aequaliform, twenty tortiform, seventeen falciform, seventy-six graciliform, sixteen truncatiform, fourteen arcuatiform elements.

Family PRIONIODINIDAE Bassler, 1925

Genus OULODUS Branson and Mehl, 1933

Type species. Oulodus mediocris Branson and Mehl, 1933.

Diagnosis. Apparatus consists of $\mathrm{Sa}, \mathrm{Sb}, \mathrm{Sc}, \mathrm{M}$, $\mathrm{Pb}$, and $\mathrm{Pa}$ elements. Denticles are round in cross section, peg-like, widely separated, very large relative to element size, and are often strongly curved. See Wang and Aldridge (2010) for detailed description of the apparatus of this genus.

Oulodus cf. petilus pacificus Savage, 1985

Figure 8.14, 8.16-17

*1985 Oulodus petilus pacificus; Savage (multielement).
Description. Sb element digyrate. Inner lateral process extends slightly down and curves back towards the cusp. Outer lateral process slightly curved and extends away from the cusp. Cusp prominent, round to slightly oval in cross section. Denticles are widely spaced, uneven in size, and slightly oval in cross section. Basal cavity large and expanded posteriorly under cusp. Sc element bipennate with anterior process sharply curved inwards and down. Posterior process extends straight behind cusp. Both processes denticulate, with unevenly sized, evenly spaced denticles that are slightly oval in cross section. Cusp prominent and oval in cross section. $\mathrm{Pb}$ element digyrate. Outer lateral process fairly straight and extends sharply down from cusp. Inner lateral process curved away from cusp. Denticles and cusp slightly oval in cross section.

Remarks. The extended processes and widely spaced denticles on the Sc element, and the geometry of the processes on the $\mathrm{Pb}$ element are comparable to Oulodus petilus pacificus Savage (1985, figure 11G-H, 11C-D). The curvature of the lateral processes of the $\mathrm{Sb}$ element (Figure 8.16) is similar to the specimen shown in figure $111-J$ in Savage (1985), but the denticles are not oriented outwardly as much as that shown in Savage (1985). One difference of all the specimens with those illustrated in Savage (1985) is that the denticles are slightly more circular in cross section.

Material examined. One $\mathrm{Sb}$, one $\mathrm{Sc}$, one $\mathrm{Pb}$ element.

Oulodus sp. indet.

Figure 8.13, 8.15

Description. Sa element alate, with a large, posteriorly reclined cusp. One lateral process broken, other extends downward and away from cusp. Lateral process has three widely separated, peg-like denticles, with a U-shaped profile between them. Basal cavity wide and slightly expanded posteriorly. $\mathrm{Pa}$ ? element digyrate, with slightly twisted lateral processes. Cusp reclines anteriorly over expanded basal cavity.

Remarks. Numerous specimens were recovered that exhibit the widely spaced, peg-like denticles, and expanded basal cavities that are diagnostic features of Oulodus, but were either not elements useful for species diagnosis or too poorly preserved to assign to a species.

Material examined. Four Sa, five $\mathrm{Sc}$, one $\mathrm{Pb}$, one $\mathrm{Pa}$.

Genus PSEUDOLONCHODINA Zhou, Zhai, and Xian, 1981 
1981 Neoplectospathodus; Zhou, Zhai, and Xian, p. 134.

1985 Aspelundia Savage, p. 725.

1990 Aspelundia Savage; Armstrong, emended diagnosis, p. 49.

2010 Pseudolonchodina; Wang and Aldridge, p. 80.

Type species. Pseudolonchodina irregularis Zhou, Zhai, and Xian, 1981.

Diagnosis. Apparatus is composed of $\mathrm{Sa}, \mathrm{Sb}, \mathrm{Sc}$, $\mathrm{M}, \mathrm{Pa}$, and $\mathrm{Pb}$ elements. $\mathrm{Pa}$ and $\mathrm{Pb}$ elements have a central cusp with two lateral processes. Lateral processes twist at different angles away from the cusp, giving the elements a digyrate morphology. $\mathrm{M}$ elements are dolabrate with a basal cavity that extends the length of the process. Sa elements are alate with a basal cavity that extends posterior to the cusp. Sb elements are tertiopedate with a deep basal cavity beneath each process. Sc elements are bipennate. Denticles are long, thin, and laterally compressed.

Remarks. Aspelundia is a junior synonym of Pseudolonchodina. See Wang and Aldridge (2010) for discussion.

\section{Pseudolonchodina sp. $\mathrm{n}$.}

Figure 8.1-12, 8.19

Description. Sa elements alate. Lateral processes denticulate and recurved in aboral view. Denticles closely spaced, evenly sized, compressed, and slightly curved posteriorly. Cusp reclines at a sharper angle than denticles. Highly compressed basal cavity extended posterior to the cusp. Sb elements tertiopedate. Denticulate lateral processes extend down from the cusp at slightly different angles. Denticulate, compressed posterior process inclined towards one of the lateral process. Basal cavity compressed, and extends along most of the lateral processes and along the posterior process. $\mathrm{Sc}$ elements bipennate. Anterior process denticulate and angled inwards. Denticulate posterior process extends straight behind cusp. $M$ elements have a large posteriorly reclined and inwardly inclined cusp. Posterior process bowed with five to seven denticles. Basal cavity bowed in with the same geometry as the posterior process. $\mathrm{Pb}$ elements digyrate. Lateral processes twisted and bowed to different degrees in different specimens. Outer lateral process extends slightly down and away from cusp. Inner lateral process extends away from cusp. Denticles are compressed, closely spaced, and slightly curved posteriorly. $\mathrm{Pa}$ elements digyrate. Lateral processes extend away from cusp at slightly different angles. Processes not as twisted and bowed as those on $\mathrm{Pb}$ elements. Basal cavity compressed and extended along lateral processes. In most of the specimens recovered, white matter extends down below the denticles and is separated from the white matter of adjacent denticles by translucent material. Denticles are not fused in most speciemens for all element types. In a minority of $\mathrm{M}$ and Sc element specimens, the denticles farthest away from the cusp are slightly fused (e.g., Figure 8.7, 8.10).

Remarks. The Sc elements are almost indistinguishable from those of Wurmiella polinclinata. Some of the elements identified as occupying the Sc position of Pseudolonchodina sp. n. may actually be juvenile $S_{3 / 4}$ elements of Wurmiella? polinclinata, which could be a reason for the overrepresentation of Pseudolonchodina Sc elements.

The general shape and many of the characteristics of our specimens are similar to specimens classified as Pseudolonchodina fluegeli (Walliser, 1964). However, the discrete (non-fused) denticles on our $\mathrm{Pa}$ specimens provides a consistent character for distinguishing our specimens from the wide range of morphologies attributed to Psl. fluegeli (Walliser).

The species concept of Psl. fluegeli is extremely broad, partially due to a lack of a multielement apparatus reconstruction using Walliser's type material (Wang and Aldridge, 2010). There have been numerous reconstructions of fluegeli by various authors (e.g., Aldridge, 1979; Savage, 1985; Bischoff, 1986; Over and Chatterton, 1987; Armstrong, 1990), each using specimens from different regions and chronostratigraphic levels. The apparatus reconstructions and element descriptions of Bischoff (1986; as Oulodus planus planus) and Armstrong (1990; as Aspelundia fluegeli) are the most complete, and the most widely followed in subsequent conodont research (e.g., Wang and Aldridge, 2010). The position of elements in their apparatus reconstructions are similar, but their $\mathrm{Pa}$ and $\mathrm{Pb}$ elements are switched, and Armstrong added a bipennate $\mathrm{Sb}$ element to the apparatus (termed $\mathrm{Sb}_{2}$ ) and suggested that the apparatus might also include an Sd element (Armstrong, 1990, p. 53-54). There are also differences between Bischoff (1986) and Armstrong (1990) in the degree of fusion of the denticles on most element types. Bischoff (1986) describes the denticles on $\mathrm{Pb}, \mathrm{M}$, and $\mathrm{Sb}$ as basally confluent (slightly fused), denticles on Sa and Sc as discrete, and denticles on $\mathrm{Pa}$ elements as fused to mid-height. In contrast, Armstrong (1990) describes denticles as partially fused on $\mathrm{Pb}$ (= $\mathrm{Pa}$ of Bischoff, 1986) ele- 
ments, and fused to mid-height on $\mathrm{M}$ elements and the anterior process of Sc elements.

Some attempts have been made to distinguish different morphologies of Psl. fluegeli s.l. as subspecies. Bischoff (1986) erected Oulodus planus planus and the stratigraphically higher Oulodus planus borenorensis based on characteristics of $\mathrm{Sb}$ and $\mathrm{Sc}$ elements. The $\mathrm{Sb}$ elements of $\mathrm{Ou}$. planus borenorensis have a shortened lateral process bearing one large and one or two small denticles. The Sc element of Ou. planus borenorensis is characterized by the presence of an elongated anterior process on Sc elements bearing 8-11 tall denticles and a posterior process with posteriorlyreclined denticles that increase in size away from the cusp.

Männik (2007a) separated Aspelundia? fluegeli ssp. n. from Asp? fluegeli fluegeli based primarily on the height and fusion of denticles, and the distribution of white matter below the denticles. Asp? fluegeli ssp. $\mathrm{n}$. is characterized by tall, thin denticles that are fused up to mid-height, whereas the denticles of Asp? fluegeli fluegeli are wider and more rubust, and fused in their lower halves (Männik, 2007, p. 57; Männik, personal commun., 2016). The white matter below the denticles of Asp? fluegeli ssp. $\mathrm{n}$. is separated by rectangular blocks of translucent material, and the white matter of Asp? fluegeli fluegeli is only separated by thin strips of translucent material (Männik, 2007a, figure 10). The fusion of the denticles in both subspecies of Asp? fluegeli sensu Männik is very different from that of Bischoff (1986) and Wang and Aldridge (2010), where the denticles of all element types except for $\mathrm{Pa}$ elements are only slightly fused or discrete. Asp? fluegeli fluegeli sensu Männik is somewhat more similar to the specimens described in Armstrong (1990), which contains elements with denticles fused to mid-height. However, to further muddy the taxonomic waters of this species, the Sa and $\mathrm{Sb}$ elements of Asp? fluegeli sensu Männik do not have posterior processes (Männik, personal commun., 2016). The Sa and Sb elements of Bischoff (1986), and the $\mathrm{Sa}$ and $\mathrm{Sb}_{1}$ elements of Armstrong (1990) are tertiopedate, thus making it likely that Asp? fluegeli sensu Männik is a separate taxon from Psl. fluegeli s.l. altogether.

Material examined. Eight Sa, twelve Sb, thirty Sc, twelve $\mathrm{M}$, ten $\mathrm{Pb}$, two $\mathrm{Pa}$ elements.

\section{Family PSEUDOONEOTODIDAE Wang and Aldridge, 2010 \\ Genus PSEUDOONEOTODUS Drygant, 1974}

Type species. Oneotodus? beckmanni Bischoff and Sannemann, 1958.

Diagnosis. Apparatus consists of squat, conical elements with one to three nodes, small denticles, or short ridges on the upper surface. Basal cavities are circular to slightly triangular and highly excavated, giving the entire element very thin walls.

\section{Pseudooneotodus beckmanni (Bischoff and Sannemann, 1958) \\ Figure 7.6}

*1958 Oneotodus? beckmanni; Bischoff and Sannemann, pl. 15, figs. 22-25.

2007 Psuedooneotodus beckmanni Bischoff and Sannemann; Corradini, pl. 1, figs. 1-7 (synonymy through 2006).

2007b Psuedooneotodus beckmanni Bischoff and Sannemann; Zhang and Barns, fig. 10.5.

2010 Psuedooneotodus beckmanni Bischoff and Sannemann; Wang and Aldridge, pl. 2, figs. 1926.

2011 Psuedooneotodus beckmanni Bischoff and Sannemann; Zhang, fig. 17.7.

2013 Psuedooneotodus beckmanni Bischoff and Sannemann; Sumrall, Heredia, Rodríguez, and Mestre, fig. 4F.

2014 Psuedooneotodus beckmanni Bischoff and Sannemann; Zhang and Pell, fig. 6.8.

2015 Psuedooneotodus beckmanni Bischoff and Sannemann; Jarochowska and Munnecke, fig. $7 \mathrm{H}-\mathrm{I}$.

2016 Psuedooneotodus beckmanni Bischoff and Sannemann; Waid and Over, fig. 3.27.

Description. Elements conical, squat, with one node at the tip of the element. Basal cavities circular or slightly oval. White matter restricted to node and just below the node.

Material examined. Three elements.

Family SPATHOGNATHODONTIDAE Hass, 1959 Genus WURMIELLA Murphy, Velenzuela-Ríos, and Carls, 2004

Type species. Ozarkodina excavata tuma Murphy and Matti, 1983.

2004 Wurmiella tuma Murphy and Matti; Murphy, Velenzuela-Ríos, and Carls, p. 11.

Diagnosis. Apparatus consists of one unpaired and five paired elements $\left(S_{0}, S_{1 / 2}, S_{3 / 4}, M, P_{1}\right.$, and $P_{2}$ ). $P_{1}$ elements blade-like, with denticles fairly uniform in size. $P_{1}$ elements have a small basal cavity that slightly flares outwards into small lobes lacking ornamentation. $\mathrm{P}_{2}$ elements angulate to slightly angulate with a cusp much larger than denticles on anterior and posterior processes. The posterior process on both the $P_{1}$ and $P_{2}$ elements 
can be slightly deflected laterally. See Murphy et al. (2004) for thorough discussion of the distinguishing characteristics of Wurmiella.

Remarks. During the transition from form-taxonomy to multielement taxonomy, most of the species in genus Spathognathodus were transferred to Ozarkodina, regardless of distinct variations in the morphology of $P$ elements (see Valenzuela-Ríos and Murphy, 1997). This allowed for a generic concept that included very diverse morphologies, and likely included numerous species not closely related. Murphy et al. (2004) restrict Ozarkodina to taxa similar to Ozarkodina typica Branson and Mehl, 1933. The genus Wurmiella was erected by Murphy et al. (2004) to include spathognathodid taxa that have $P_{1}$ elements with small, unornamented basal platforms similar to $O$. typica, but which have relatively evenly-sized denticles, and lack a sail-like fan of large denticles at the anterior end of the element. According to Murphy et al. (2004), $\mathrm{P}_{2}$ and $\mathrm{M}$ element morphologies of Wurmiella are fairly stable throughout the range of the genus, and are distinct enough from the $\mathrm{P}_{2}$ and $\mathrm{M}$ morphologies of Ozarkodina to be considered the most useful for distinguishing species of the two genera.

\section{Wurmiella? polinclinata polinclinata (Nicoll and Rexroad, 1968)}

Figures 9.1-9.8, 11.1-12, 11.14

*1968 Spathognathodus polinclinatus; Nicoll and Rexroad, pl. 2, figs. 19-20 ( $P_{1}$ element).

1992 Ozarkodina polinclinata polinclinata Nicoll and Rexroad; Männik, figs. 4.1-4.28, 5.1-5.7, pl. figs. 8, 10-20 (multi-element; synonymy of $\mathrm{P}_{1}$ elements through 1992).

1994 Ozarkodina polinclinata Nicoll and Rexroad; Kleffner, fig. 10.1.

2005 Ozarkodina polinclinata polinclinata Nicoll and Rexroad; Metzger, pl. 1, fig. 24.

2006 Ozarkodina polinclinata polinclinata Nicoll and Rexroad; Hints, Killing, Männik, and Nestor, pl. 3, fig. 5.

2007a Ozarkodina polinclinata polinclinata Nicoll and Rexroad; Männik, fig. 91.

2007b Ozarkodina polinclinata Nicoll and Rexroad; Zhang and Barnes, fig. 12.30-12.31.

Description. $\mathrm{P}_{1}$ elements blade-like with an identifiable cusp. Denticles along the anterior part oriented upwards and mostly evenly sized. In some specimens the most anterior denticles may be taller and wider, and rarely, the anterior-most two or three denticles may be completely fused. Denticles over basal cavity larger and reclined posteri- orly. Denticles are partially fused creating grooves between each denticle. Some grooves extend almost to base of element. Aboral margin anterior to basal cavity straight to slightly curved. Basal cavity flares out into a small, unornamented basal platform. Basal platforms restricted to the posterior one third of the total length of the blade. Blade posterior to cusp deflected slightly inwards. $P_{2}$ elements slightly angulate with a posteriorly reclined cusp that is much larger than the other denticles. Denticles on anterior process oriented upwards to slightly reclined posteriorly. Denticles on posterior process reclined posteriorly at the same angle as the cusp. Posterior process deflected slightly inwards. $M$ element dolabrate, with a slightly twisted posterior process extending straight behind cusp. Basal cavity widest under cusp and extends along posterior process. $\mathrm{S}_{0}$ elements alate, with closely spaced compressed denticles. Basal cavity small. $S_{1 / 2}$ elements have a central cusp with two lateral processes extending down and away at different angles. Inner lateral process slightly wider than outer. Denticles closely spaced and triangular. $\mathrm{S}_{3 / 4}$ elements bipennate. Posterior process extends straight behind the cusp. Anterior process inclined inwards. Denticles closely spaced, short, and triangular.

Remarks. The $M$ elements are very similar to those of Psl. fluegeli, but can be distinguished by their narrower basal cavities and slightly straighter posterior process.

Murphy et al. (2004) reassigned polinclinata from genus Ozarkodina to Wurmiella without elaboration. We tentatively agree with this assignment, because the $P_{2}$ elements exhibit the slight asymmetry in the basal platform that characterizes species of Wurmiella. The $\mathrm{P}_{1}$ elements, however, differ considerably from the morphology of the type species of Wurmiella, as well as the diagnosis of Murphy et al. (2004), page 8, who state that Wurmiella is a "...genus whose species have a sexmembrate apparatus characterized primitively by processes without strong variation of adjacent denticles." The denticles on $W$. ? polinclinata can greatly vary in size on some specimens, particularly those adjacent to the cusp and at the posterior end of the blade (Figures 9.7, 9.8, 11.1, 11.2), in contrast to the very evenly-sized and oriented denticles on Wurmiella tuma (Murphy et al., 2004, figure 2.1619).

Transitional morphologies absent in the Baltic between Wurmiella? polinclinata estonica Männik (1992; see below) and $W$.? polinclinata polinclinata may be present in the specimens from the Garrison 
Core. Männik (1992) differentiated the stratigraphically lower $W . ? p$. estonica from the higher $W . ? p$. polinclinata based on the character of the denticulation of $\mathrm{P}_{1}$ elements. Most W.? p. estonica specimens lack a cusp, have denticles that are uniformly sized, completely fused, oriented straight to very slightly reclined anteriorly at the anterior end of the blade and posteriorly at the posterior end, and lack a cusp (Männik, 1992, figure 3). Wurmiella? $p$. polinclinata, in contrast, has a cusp, the denticles vary in size, are only slightly fused to not fused, and progressively recline more posteriorly closer to the posterior end of the blade. Specimens from 135.65-136.10 m (445.66-446.53ft) in the Garrison Core (Figure 11.15-17) can be confidently assigned to W.? p. estonica. Two from GC 134.06$131.31 \mathrm{~m}$ (439.83-440.66ft; Figure 11.12, 11.14) show some characteristics of both subspecies. The characteristics of $W$.? $p$. estonica are that the denticles on each of the specimens recline slightly, if at all, and the denticles are fused with the exception of the most posterior two denticles. The characteristics of $W$.? $p$. polinclinata shown by the specimens are the relatively large size difference between the anterior and posterior denticles, and the development of a cusp. The $W$.? polinclinata polinclinata specimens illustrated in Männik (1992) show much more morphological variability than those illustrated as W.? polinclinata estonica, so we suggest that specimens that do not conform strictly to the diagnosis of $W . ? p$. estonica be assigned to $W$.? polinclinata polinclinata.

Material examined. Seven $S_{0}$, fourteen $S_{1 / 2}$, six $S_{3 / 4}$, two $M$, eighteen $P_{2}$, twenty-one $P_{1}$ elements.

Wurmiella? polinclinata estonica Männik, 1992

Figure 11.13, 11.15-17

1987 Ozarkodina polinclinata; Nicoll and Rexroad; Kleffner, fig. 5.17-5.22 (multielement).

1991 Ozarkodina polinclinata Nicoll and Rexroad; Kleffner, fig. 6.17.

*1992 Ozarkodina polinclinata estonica Männik; Männik, fig. 3.1-3.30, 5.8-5.29; pl. figs. 1-7, 9 (multielement; synonymy through 1992).

2002 Ozarkodina polinclinata estonica Männik; Männik, fig. 7A-B, D.

2007 Ozarkodina polinclinata estonica Männik; Loydell, Kleffner, Mullins, Butcher, Matteson, and Ebert, fig. 6ff, mm.

2008 Ozarkodina polinclinata estonica Männik; Männik, fig. 8K.

2009 Ozarkodina polinclinata estonica Männik; Munnecke and Männik, fig. $5 \mathrm{H}$.

2014 Ozarkodina polinclinata estonica Männik; Sullivan, Kleffner, and Brett, fig. 5.7-5.8.
2015 Ozarkodina polinclinata estonica Männik; Bancroft, Brunton, and Kleffner, fig. 5A.

Description. $S$ and $M$ elements identical to those from $W$. $p$. polinclinata. $\mathrm{P}_{1}$ elements blade-like. Denticles evenly sized and spaced, and fused almost to their tips. Denticles straight to slightly reclined posteriorly in the posterior portion of the element. Aboral margin curved to slightly recurved. Basal cavity flared outward, forming small, arched, and unornamented basal platform.

Remarks. See remarks for Wurmiella? polinclinata polinclinata for discussion.

Material examined. Three $S_{0}$, three $S_{1 / 2}$, one $S_{3 / 4}$, three $M$, three $P_{2}$, three $P_{1}$ elements.

\section{DISCUSSION}

\section{Biozonation of the LaPorte City Formation}

Conodonts representative of the Pterospathodus eopennatus Superzone, the Pt. eopennatus ssp. n. 2 Zone, and the Pt. amorphognathoides angulatus Zone were recovered from the LPC in the Garrison Core (Figure 5). The lower boundary of the Pt. eopennatus Superzone is tentatively placed at the first occurrence of $S$ elements assigned to Wurmiella? polinclinata estonica (GC 137.12-137.40 m; 449.87-450.79ft), because that species first appears just below the base of the Pt. eopennatus ssp. n. 1 Zone (Männik, 2007a). The first occurrence of $W$. ? $p$. estonica $P_{1}$ elements was at GC 135.65-136.10 m (445.06-446.53ft). Morph $2 \mathrm{~b}$ of Pt. eopennatus was found at GC 131.95-132.23 m (432.92-433.84ft). The base of the Pt. eopennatus ssp. n. 2 Zone is placed here, because morph $2 \mathrm{~b}$ is assigned to that subspecies (Männik, 1998). The base of the Pt. amorphognathoides angulatus Zone is placed at the first occurrence of a $\mathrm{Pb}_{1}$ element of $P t$. am. angulatus. This specimen was originally identified as Pt. am. amorphognathoides in Metzger (2005), but it compares much more closely with $\mathrm{Pb}_{1}$ elements of $P t$. am. angulatus (Figure 10.4-5; see Systematic Paleontology). The extent of the Pt. am. angulatus Zone at the top of the LPC in the GC is unclear. All of the specimens from the GC in this study that are assigned to Pt. am. angulatus are poorly preserved, and no specimens diagnostic of higher biozones were found at the top of the core.

Conodonts representative of the Pterospathodus eopennatus Superzone, Pt. eopennatus ssp. n. 2 Zone, and Pt. amorphognathoides angulatus Zone were recovered from the DX5 outcrop of the LPC (Figure 6). The lowermost sample (DX5 0.04$0.08 \mathrm{~m}$ ), in the BCQ member of the Scotch Grove 
Formation, yielded two poorly preserved $\mathrm{P}_{1}$ specimens tentatively identified as Aulacognathus bullatus. The first occurrence of this species is within the Pt. am. angulatus Zone in the Baltic, but occurs much lower in other basins. In the Northwest Territories of Canada, Over and Chatterton (1987) recovered $A$. bullatus with Astropentagnathus irregularis Mostler (1967), which is restricted to approximately the Pt. eopennatus ssp. n. 1 Zone (Männik, 2007a). The base of the Pt. eopennatus ssp. n. 2 Zone is placed at approximately $0.5 \mathrm{~m}$ in the section, at the first occurrence of the nominal subspecies (reported as sample interval DLH 4 in Metzger, 2005; Figure 4.1). The base of the Pt. am. angulatus Zone is placed at approximately $1.1 \mathrm{~m}$ in the section, at the first occurrence of the nominal subspecies (sample DLH 6, plate 1, figures 8, 16, 17 in Metzger, 2005; Figure 4.7-8). Specimens of Pt. eopennatus ssp. n. 2 sensu Männik (1998; Figure 4.2-3) were reported from this sample by Metzger (2005). This does not indicate that the ranges of Pt. eopennatus and Pt. am. angulatus overlapped, because the boundary between the two could be within the sample interval.

Metzger (2005) reported elements of Pterospathodus eopennatus in sample intervals DLH 8 , 9 , and 11-all of which are well above the first occurrence of Pt. am. angulatus. Two S elements were reported in DLH 8, but no $S$ elements assignable to genus Pterospathodus were present in the microfossil slide from that interval. Two specimens similar to $\mathrm{M}$ elements of Pterospathodus were found, but they are not diagnostic of species within the genus. Metzger (2005) reported one $\mathrm{Pa}$ and $\mathrm{Pb}$ element of Pt. eopennatus in DLH 9 (Figure 4.5 and 4.6). The $\mathrm{Pa}$ element is most similar to Pt. eopennatus, but the $\mathrm{Pb}$ element is more similar to those of Pt. am. angulatus (e.g., Männik, 1998, text-figures $8 \mathrm{~T}, 8 \mathrm{U})$. Metzger reported a $\mathrm{Pb}$ element of Pt. eopennatus in DLH 11 (Figure 4.10). The curvature of the aboral margin of the element, angle of the lateral process, and distribution of white matter below each denticle are much more similar to that of $\mathrm{P}_{2}$ element of the genus Aulacognathus (c.f. Figure 9.11). It is tentatively assigned to Aulacognathus bullatus. No additional evidence was found verifying the stratigraphic extent of the coexistence of Pt. eopennatus and Pt. am. angulatus reported by Metzger (2005). Due to the possibility of specimen reworking or sample contamination, there is not enough evidence to extend the range of Pt. eopennatus up or Pt. am. angulatus down. Additionally, some of the younger Pa morphotypes of Pt. eopennatus can be almost indistinguishable from older Pa elements of Pt. am. angulatus, so $\mathrm{Pb}_{2}$ and $\mathrm{S}$ elements are used to distinguish between the species (Männik, personal commun., 2016). Few $\mathrm{Pb}_{2}$ or $\mathrm{S}$ elements were recovered from the DX5 outcrop, so it is possible that the specimens identified as Pt. eopennatus may actually be $P t$. am. angulatus.

\section{Comparison with the Baltic}

The stratigraphic ranges of two taxa are different in the East-Central lowa Basin than in the Baltic Basin. If the specimen we tentatively identify as Astrolecignathus milleri? is actually Ast. milleri, then its stratigraphic range is slightly higher in the East-Central lowa Basin than in the Northwest Territories, where Over and Chatterton (1987) recovered it with specimens diagnostic of the Pt. eopennatus ssp. n. 1 Zone. It was recovered from a sample above the last occurrence of Aulacognathus kuehni within the Upper Pteropathodus eopennatus ssp. n. 2 Subzone, and close to the base of the Pt. amorphoghanoides angulatus Zone (Figure 5). In the Baltic, a species identified as Apsidognathus milleri occurs from the Pt. eopennatus ssp. n. 1 Zone to the Lower Pt. eopennatus ssp. n. 2 Subzone (Männik, 2007a). Männik (2007a) reclassified Ast. milleri as Apsidognathus milleri without any elaboration. We disagree with this classification, and maintain that the species should remain in Astrolecignathus (see Systematic Paleontology). Furthermore, the illustrated specimens identified as Aps. milleri in Männik (2007a, 2008) have some key differences with the holoand paratype specimens in Over and Chatterton (1987), and may be a separate species belonging to the genus Apsidognathus.

A new species of Psuedolonchodina described from the LaPorte City limestone is similar to, but has some characteristics distinct from both Aspelundia? fluegeli fluegeli sensu (Männik, 2007a) and Aspelundia? fluegeli ssp. n. Männik (2007a). The narrow, tall denticles of Psl. sp. n. are similar to Asp? ssp. n., but do not have denticles that are fused to mid-height, as they are in Asp? ssp. n. The white matter below the denticles of Psl. sp. n. specimens is more similar to Asp? fluegeli fluegeli, but the denticles are not as robust or fused. See Systematic Paleontology for further discussion.

In the Baltic, Wurmiella? polinclinata estonica ranges from just below the base of the Pterospathodus eopennatus ssp. $\mathrm{n}$. 1 Zone to the base of the Pt. amorphognathoides lithuanicus Zone (Männik, 2007a, figures 4), and the first occurrence 
of W.? p. polinclinata occurs at, or just below the base of the Pt. am. amorphognathoides Zone (Figure 1). In the LPC of the GC, specimens fitting the diagnosis and illustrated specimens of W.? $p$. polinclinata (see Systematic Paleontology for discussion) occur within the Pt. eopennatus ssp. n. 2 Zone. In the DX5 outcrop section, $\mathrm{P}_{1}$ elements of W.? p. polinclinata occur within the Pt. am. angulatus Zone. This means that the first appearance of $W$. p p polinclinata is much lower than previously identified, and the subspecies cannot be used as an index fossil for the Pt. am. amorphognathoides Zone in global correlations. The separation of $W$.? polinclinata into stratigraphically distinct subspecies $W$.? polinclinata estonica and $W$. ? $p$. polinclinata is still justifiable on a regional scale, because the stratigraphic ranges of the two morphologies have not been shown to co-occur over more than one sample interval in this or any other study published to date. It appears likely that W.? $p$. polinclinata evolved from a restricted $W$.? polinclinata estonica population in the N. A. Midcontinent first, and physical or environmental barriers prevented migration to, and colonization of, the Baltic Basin.

\section{CONCLUSION}

Conodonts diagnostic of the Pterospathodus eopennatus Superzone, Pt. eopennatus ssp. n. 2 Zone, and Pt. amorphognathoides angulatus Zone were recovered from the LaPorte City Formation of eastern lowa. The identification of these zones represents the highest resolution Silurian conodont zonation to date from the North American Midcontinent, and permits direct comparison of the ranges of conodont species to those in Baltica. A new species of Psuedolonchodina similar to Psl. fluegeli sensu Bischoff (1986) was recorded from the Pt. eopennatus Superzone through the Pt. amorph. angulatus Zone. They are distinguished from Psl. fluegeli by the absence of fused denticles on $\mathrm{Pa}$ elements and discrete or nearly discrete denticles on the other elements. The specimens were substantially different from Aspelundia fluegeli fluegeli sensu Männik (2007a). The first occurrence of Wurmiella? polinclinata polinclinata is in the Pt. eopennatus ssp. n. 2 Zone in the LaPorte City Formation, which is significantly lower than its first occurrence in the Pt. am. amorphognathoides Zone in the Baltic. The extended range of $W$.? polinclinata polinclinata means it cannot be used as an index fossil diagnostic of the Pt. am. amorphognathoides Zone. This study highlights the importance of continued biostratigraphic research towards refining biostratigraphic zonations and showcases the usefulness of the limestone units of the northern Midcontinent for refining global conodont zonation.

\section{ACKNOWLEDGMENTS}

We would like to thank N.E.B. McAdams and A.M. Bancroft for their assistance in the field, and J. Adrain for providing the photography equipment used in the study. B.J. Witzke was very helpful in getting this project off the ground, and E. Finzel and B.J. Witzke provided helpful initial feedback on the manuscript. The thorough and thoughtful reviews of M.A. Kleffner and P. Männik are greatly appreciated. We would also like to thank the lowa Geological Survey for providing the core used in this study. This work was supported by NSF grant \# CAREER-1455030 to B.D. Cramer and a GSA Foundation Graduate Research Grant to C.B. Waid. This is a contribution to the International Geoscience Programme (IGCP) Project 591 - The Early to Middle Paleozoic Revolution.

\section{REFERENCES}

Aldridge, R. 1972. Llandovery conodonts from the Welsh Borderland. Bulletin of the British Museum-Geology, 22:1-106.

Aldridge, R. 1975. The stratigraphic distribution of conodonts in the British Silurian. Journal of the Geological Society, 131:607-618.

Aldridge, R. 1979. An upper Llandovery conodont fauna from Pear Land, eastern North Greenland. Rapport Grønlands Geologiske Undersøgelse, 91:7-23.

Armstrong, H. 1990. Conodonts from the Upper Ordovician - Lower Silurian carbonate platform of North Greenland. Grønlands Geologiske Undersøgelse, Bulletin 159. Grønlands Geologiske Undersøgelse, Copenhagen.

Barnes, C. 1977. Ordovician conodonts from the Ship Point and Bad Cache Rapids Formations, Melville Peninsula, Southeastern District of Franklin. Geological Survey of Canada Bulletin, 269:99-119.

Bassler, R. 1925. Classification and stratigraphic use of the conodonts. Geological Society of America Bulletin, 36:218-220.

Bauer, J. 1994. Conodonts from the Bromide Formation (Middle Ordovician), South-Central Oklahoma. Journal of Paleontology, 68: 358-376.

Bischoff, G. 1986. Early and Middle Silurian conodonts from midwestern New South Wales. Courier Forschungsinstitut Senckenberg, 89:1-269.

Bischoff, G. and Sannemann, D. 1958. Unterdevonische conodonten aus dem Frankenwald Notizblatt des Hessisches Landesamt für Bodenforschung zu Wisbaden, 86:87-110. 
Branson, E. and Branson, C. 1947. Lower Silurian conodonts from Kentucky. Journal of Paleontology, 21:549-556.

Branson, E. and Mehl, M. 1933a. Conodonts from the Bainbridge (Silurian) of Missouri. University of Missouri Studies, 8:39-52.

Branson, E. and Mehl, M. 1933b. Conodonts from the Plattin (Middle Ordovician) of Missouri. University of Missouri Studies, 8:101-119.

Brett, C., Baarli, B., Chowns, T., Cotter, E., Driese, S., Goodman, W., and Johnson, M. 1998. Early Silurian condensed intervals, ironstones, and sequence stratigraphy in the Appalachian Foreland Basin, p. 89143. In Landing, E. and Johnson, M. (eds.), Silurian Cycles: Linkages of Dynamic Stratigraphy with Atmospheric, Oceanic, and Tectonic Changes. New York State Museum Bulletin, 491. New York State Museum, Albany, New York.

Calner, M. 2008. Silurian global events - at the tipping point of climate change, p. 21-58. In Elewa, A. (ed.), Mass Extinctions. Springer-Verlag, Heidelberg.

Calner, M., Eriksson, M., Clarkson, E., and Jeppsson, L. 2008. An atypical intra-platform environment and biota from the Silurian of Gotland, Sweden. GFF, 130:79-86.

Calner, M. and Jeppsson, L. 2003. Carbonate platform evolution and conodont stratigraphy during the middle Silurian Mulde Event, Gotland, Sweden. Geological Magazine, 140:176-203.

Cody, R., Levy, R., Harwood, D., and Sadler, P. 2008. Thinking outside the zone: high-resolution quantitative diatom biochronology for the Antarctic Neogene. Palaeogeography, Palaeoclimatology, Palaeoecology, 260:92-121.

Cooper, R., Sadler, P., Munnecke, A., and Crampton, J. 2014. Graptoloid evolutionary rates track Ordovician - Silurian global climate change. Geological Magazine, 151:349-364.

Corradini, C. 2007. The conodont genus Pseudooneotodus Drygant from the Silurian and Lower Devonian of Sardinia and the Carnic Alps (Italy). Bollettino della Società Paleontologica Italiana, 46:139-148.

Corradini, C. and Corriga, M. 2012. A Prídolí-Lochkovian conodont zonation in Sardina and the Carnic Alps: implications for a global zonation scheme. Bulletin of Geosciences, 87:635-650.

Corriga, M. and Corradini, C. 2009. Upper Silurian and Lower Devonian conodonts from the Monte Cocco II Section (Carnic Alps, Italy). Bulletin of Geosciences, 84:155-168.

Cramer, B., Brett, C., Melchin, M., Männik, P., Kleffner, M., McLaughlin, P., Loydell, D., Munnecke, A., Jeppsson, L., Corradini, C., Brunton, F., and Saltzman, M. 2011. Revised correlation of Silurian Provincial Series of North America with global and regional chronostratigraphic units and $\delta^{13} C_{\text {carb }}$ chemostratigraphy. Lethaia, 44:185-202.
Cramer, B., Kleffner, M., Brett, C., McLaughlin, P., Jeppsson, L., Munnecke, A., and Samtleben, C. 2010. Paleobiogeography, high-resolution stratigraphy, and the future of Paleozoic biostratigraphy: Fine-scale diachroneity of the Wenlock (Silurian) conodont Kockelella walliseri. Palaeogeography, Palaeoclimatology, Palaeoecology, 294:232-241.

Cramer, B., Kleffner, M., and Saltzman, M. 2006. Chemostratigraphic correlation of the Lower Silurian deposits in eastern lowa: placing the LlandoveryWenlock boundary in the mid-continent, p. 103-109. In Day, J., Luczaj, J., and Anderson, R. (eds.), SEPM Field Guide, 67th Tri-State Field Conference, lowa Geological Survey, Guidebook Series 25. lowa Geological Survey, lowa City.

Cramer, B., Vandenbroucke, T., and Ludvigson, G. 2015. High-resolution event stratigraphy (HiRES) and the quantification of stratigraphic uncertainty: Silurian examples of the quest for precision in stratigraphy. Earth-Science Reviews, 141:136-153.

Drygant, D. 1974. Simple conodonts from the Silurian and lowermost Devonian. Palaeontologischeskii Sbornik, 10:64-70.

Epstein, A., Epstein, J., and Harris, L. 1977. Conodont Color Alteration - An Index to Organic Metamorphism, USGS Professional Paper 995. U.S. Government Printing Office, Washington.

Ethington, R. 1959. Conodonts of the Ordovician Galena Formation. Journal of Paleontology, 33:257-292.

Farrell, J. 2004. Siluro-Devonian conodonts from the Camelford Limeston, Wellington, New South Wales, Australia. Palaeontology, 47:937-982.

Hass, W. 1959. Conodonts from the Chappel Limestone of Texas. USGS Professional Paper 294-J:363-399.

Hints, O., Killing, M., Männik, P., and Nestor, V. 2006. Frequency patterns of chitinozoans, scolecodonts, and conodonts in the upper Llandovery and lower Wenlock of the Paatsalu core, western Estonia. Proceedings of the Estonian Academy of Science, Geology, 55:128-155.

Jarochowska, E. and Munnecke, A. 2015. Late Wenlock carbon isotope excursions and associated conodont fauna in the Podlasie Depression, eastern Poland: a not-so-big crisis? Geological Journal, 51(5):683-703. doi:10.1002/gj.2674

Jeppsson, L. 1997. A new latest Telychian, Sheinwoodian and early Early Homerian (Early Silurian) standard conodont zonation. Transactions of the Royal Society of Edinburgh, Earth Sciences, 88:91-114.

Jeppsson, L. and Anehus, R. 1995. A buffered formic acid technique for conodont extraction. Journal of Paleontology, 69:790-794.

Jeppsson, L. and Calner, M. 2002. Transactions of the Royal Society of Edinburgh, Earth Sciences, 93:135154.

Jeppsson, L., Eriksson, M., and Calner, M. 2006. A latest Llandovery to latest Ludlow high-resolution biostratigraphy based on the Silurian of Gotland - a summary. GFF, 128:109-114. 
Khodalevich, A. and Tschernich, V. 1973. Noveo podsemeystvo Belodellinae (Konodonty). Trudy Sverdlovskogo Gornogo Instituta, 93:42-47.

Klapper, G. 1981. Family Distomodontidae Klapper, new, p. W137. In Robison, R. (ed.), Treatise on Invertebrate Paleontology, Part W, Miscellanea, Supplement 2, Conodonta. University of Kansas Press, Lawrence, Kansas.

Klapper, G. and Murphy, M. 1974. Silurian - Lower Devonian conodont sequence in the Roberts Mountains Formation of Central Nevada. University of California Publications in Geological Sciences 111. University of California, Berkeley.

Kleffner, M. 1987. Conodonts of the Estill Shale and Bisher Formation (Silurian, southern Ohio): biostratigraphy and distribution. Ohio Journal of Science, 87:78-89.

Kleffner, M. 1991. Conodont biostratigraphy of the upper part of the Clinton Group and the Lockport Group (Silurian) in the Niagara Gorge region, New York and Ontario. Journal of Paleontology, 65:50-511.

Kleffner, M. 1994. Conodont biostratigraphy and depositional history of strata comprising the Niagaran Sequence (Silurian) in the northern part of the Cincinnati Arch region, west-central Ohio, and evolution of Kockelella walliseri (Helfrich). Journal of Paleontology, 68:141-153.

Kleffner, M. and Barrick, J. 2010. Telychian-early Sheinwoodian (Early Silurian) conodont-, graptolite-, chitanozoa-, and event-based chronostratigraphy developed using the graphic correlation method. Memoirs of the Association of Australasian Paleontologists, 39:191-210.

Loydell, D., Frỳda, J., and Gutiérrez-Marco, J.C. 2015. The Aeronian/Telychian (Llandovery, Silurian) boundary, with particular reference to sections around the El Pintado reservoir, Seville Province, Spain. Bulletin of Geosciences, 90:743-794.

Loydell, D., Kleffner, M., Mullins, G., Butcher, A., Matteson, D., and Ebert, J. 2007. The lower Williamson Shale (Silurian) of New York: a biostratigraphical enigma. Geological Magazine, 144:225-234.

Loydell, D., Männik, P., and Nestor, V. 2003. Integrated biostratigraphy of the lower Silurian of the Aizpute-41 core, Latvia. Geological Magazine, 140:205-229.

Loydell, D., Nestor, V., and Männik, P. 2010. Integrated biostratigraphy of the lower Silurian of the Kolka-54 core, Latvia. Geological Magazine, 147:253-280.

Lindström, M. 1970. A suprageneric taxonomy of the conodonts. Lethaia, 3:427-455.

Mabilard, J. and Aldridge, R. 1985. Microfossil distribution across the base of the Wenlock Series in the type area. Paleontology, 28:89-100.

Macke, D. 1995. Illinois Basin Province (064). National Assessment of United States Oil and Gas Resources - Results, Methodology, and Supporting Data. United States Geological Survey Digital Data Series DDS30, Release 2, downloaded 20 April 2015. http://certmapper.cr.usgs.gov
Männik, P. 1992. Taxonomy of the conodont species Ozarkodina polinclinata (Nicoll et Rexroad) in the Silurian of Estonia. Proceedings of the Estonian Academy of Science, Geology, 41:54-62.

Männik, P. 1998. Evolution and taxonomy of the Silurian conodont Pterospathodus. Paleontology, 41:10011050.

Männik, P. 2002. Conodonts in the Silurian of Severnaya Zemlya and Sedov archipelagos (Russia), with special reference to the genus Ozarkodina Branson and Mehl, 1933. Geodiversitas, 24:77-97.

Männik, P. 2007a. An updated Telychian (Late Llandovery, Silurian) conodont zonation based on Baltic faunas. Lethaia, 40:45-60.

Männik, P. 2007b. Recent developments in the Upper Ordovician and lower Silurian conodont biostratigraphy in Estonia. Estonian Journal of Earth Science, 56:35-46.

Männik, P. 2008. Conodont dating of some Telychian (Silurian) sections in Estonia. Estonian Journal of Earth Sciences, 57:156-169.

Männik, P., Loydell, D., Nestor, V., and Nõlvak, J. 2015. Integrated Upper Ordovician - lower Silurian biostratigraphy of the Grötlingbo-1 core section, Sweden. GFF, 137:226-244.

Männik, P., Miller, C., and Hairapetian, V. 2013. Conodonts from the Niur Formation (Silurian) of the Derenjal Mountains, Central Iran. Geological Magazine, 150:639-650.

McCracken, A. 1991. Taxonomy and biostratigraphy of Llandovery (Silurian) conodonts in the Canadian Cordillera, northern Yukon Territory. Geological Survey of Canada Bulletin, 417:65-95.

McLaughlin, P., Cramer, B., Brett, C., and Kleffner, M. 2008. Silurian high-resolution stratigraphy on the Cincinnati Arch: progress on recalibrating the layercake, p. 119-180. In Maria, A. and Counts, R. (eds.), From the Cincinnati Arch to the Illinois Basin: Geological Field Excursions along the Ohio River Valley. Geological Society of America Field Guide 12. The Geological Society of America Inc., Boulder, Colorado.

Melchin, M., Sadler, P., and Cramer, B. 2012. Chapter 21: the Silurian Period, p. 525-588. In Gradstein, F., Ogg, J., Schmitz, M., and Ogg, G. (eds.), The Geologic Time Scale 2012. Elsevier, New York City, New York.

Metzger, R. 2005. Conodont biostratigraphy of the Scotch Grove and LaPorte City formations (late Llandovery-early Wenlock; Silurian) in eastern lowa. Bulletins of American Paleontology, 369:93-104.

Mostler, H. 1967. Conodonten aus dem tieferen Silur der Kitzbühler Alpen (Tirol). Annalen des Naturhistorischen Museums in Wien, 71:295-303.

Munnecke, A., Calner, M., Harper, D., and Servais, T. 2010. Ordovician and Silurian sea-water chemistry, sea level, and climate: A synopsis. Palaeogeography, Palaeoclimatology, Palaeoecology, 296:389-413. 
Munnecke, A. and Männik, P. 2009. New biostratigraphic and chemostratigraphic data from the Chicotte Formation (Llandovery, Anticosti Island, Laurentia) compared with the Viki core (Estonia, Baltica). Estonian Journal of Earth Sciences, 58:159-169.

Murphy, M. and Matti, J. 1983. Lower Devonian conodonts (hesperius and kindlei Zones), central Nevada. University of California Publications, Geological Sciences, 123:1-82.

Murphy, M., Valenzuela-Ríos, J.I., and Carls, P. 2004. On classification of Pridoli (Silurian)-Lochkovian (Devonian) Spathognathodontidae (Conodonts). University of California, Riverside, Campus Museum Contribution, 6:1-25.

Nicoll, R. and Rexroad, C. 1968. Stratigraphy and conodont paleontology of the Salamonie Dolomite and Lee Creek Member of the Brassfield Limestone (Silurian) in Southeastern Indiana and adjacent Kentucky. Indiana Geological Survey Bulletin, 40:1-73.

Nowlan, G. 1983. Early Silurian conodonts of eastern Canada. Fossils and Strata, 15:95-110.

Nowlan, G. and Barnes, C. 1981. Late Ordovician conodonts from the Vauréal Formation, Anticosti Island, Québec. Geological Survey of Canada Bulletin, 329:1-49.

Over, D.J. and Chatterton, B. 1987. Silurian conodonts from the southern Mackenzie Mountains, Northwest Territories, Canada. Geologica et Palaeontologica, 21:1-49.

Purnell, M., Donoghue, P., Aldridge, R. 2000. Orientation and anatomical notation in conodonts. Journal of Paleontology, 74:113-122.

Pyle, L. and Barnes, C. 2003. Conodonts from a platform-to-basin transect, Lower Ordovician to lower Silurian, northeastern British Columbia, Canada. Journal of Paleontology, 77:146-171.

Rexroad, C. 1967. Stratigraphy and conodont paleontology of the Brassfield (Silurian) in the Cincinnati Arch area. Indiana Geological Survey Bulletin, 36:1-64.

Rhodes, F. 1953. Some British lower Paleozoic conodont faunas. Philosophical Transactions of the Royal Society of London, 237:261-334.

Rickards, R. Percival, I., Simpson, A., and Wright, A. 2001. Silurian biostratigraphy of the Cadia area, South of Orange, New South Wales. Proceedings of the Linnean Society of New South Wales, 123:173191.

Sansom, I. and Smith, M. 2005. Late Ordovician vertebrates from the Bighorn Mountains of Wyoming, USA. Palaeontology, 48:31-48.

Sansom, I., Armstrong, H., and Smith, M. 1994. The apparatus architecture of Panderodus and its implications for coniform conodont classification. Palaeontology, 37:781-799.

Savage, N. 1985. Silurian (Llandovery-Wenlock) conodonts from the base of the Heceta Limestone, southeastern Alaska. Canadian Journal of Earth Science, 22:711-727.
Serpagli, E. 1967. I Conodonti dell' Ordoviciano Superiore (Ashgilliano) della Alpi Carniche. Bollettino della Società Paleontologica Italiana, 6:30-111.

Smelror, M., Cocks, L.R., Mørk, A., Neuman, B., and Nakrem, H. 1997. Upper Ordovician - Lower Silurian strata and biota from offshore South Norway. Norsk Geologisk Tidsskrift, 77:251-268.

Stauffer, C. 1940. Conodonts from the Devonian and associated clays of Minnesota. Journal of Paleontology, 14:125-168.

Sullivan, N., Kleffner, M., and Brett, C. 2014. Conodont biostratigraphy, paleoecology, and taphonomy of the Second Creek Bed and Wolcott Furnace Hematite (Clinton Group) in West Central New York State. Palaeontologia Electronica17.1.10A:1-19 palaeoelectronica.org/content/2014/679-conodonts-in-second-creek-bed

Sumrall, C., Heredia, S., Rodríguez, C., and Mestre, A. 2013. The first report of South American Edrioasteroids and the paleontology and ontogeny of Rhenopyrgid Echinoderms. Acta Palaeontologica Polonica, 54:763-776.

Sweet, W. and Schönlaub, H. 1975. Conodonts of the genus Oulodus Branson and Mehl, 1933. Geologica et Palaeontologica, 9:41-59.

Sweet, W., Thompson, T., and Satterfield, I. 1975. Conodont stratigraphy of the Cape Limestone (Maysvillian) of eastern Missouri. Missouri Geological Survey Report of Investigations, 57:1-60.

Szaniawski, H. 2009. The earliest known venomous animals recognized among conodonts. Acta Palaeontologica Polonica, 54:669-676.

Torsvik, T., Smethurst, M., Meert, J., Van der Voo, R., McKerrow, W., Brasier, M., Sturt, B., and Walderhaug, H. 1996. Continental break-up and collision in the Neoproterozoic and Palaeozoic - A tale of Baltica and Laurentia: Earth-Science Reviews, 40:229-258.

Valenzuela-Ríos, J.I. and Murphy, M. 1997. A new zonation of middle Lochkovian (Lower Devonian) conodonts and evolution of Flajsella n. gen. (Conodonta), p. 131-144. In Klapper, G., Murphy, M., and Talent, J. (eds.), Paleozoic Sequence Stratigraphy, Biostratigraphy, and Biogeography: Studies in Honor of $J$. Granville ("Jess") Johnson, Geological Society of America Special Paper, 321. The Geological Society of America Inc., Boulder, Colorado.

Waid, C. and Over, D.J. 2016. Aeronian (Llandovery, Silurian) conodonts from the Densmore Creek Phosphate Bed and the Budd Road Phosphate Bed, Clinton Group, western New York State. Canadian Journal of Earth Science, 53:1-6. doi:10.1139/cjes2015-0140

Walliser, O. 1957. Conodonten aus dem oberen Gotlandium Deutschlands und der Karnischen Alpen. Notizblatt des Hessischen Landesamtes fur Bodenforschung, 85:28-52

Walliser, O. 1964. Conodonten des Silurs. Abhandlungen des Hessischen Landesamtes für Bodenforschung, 41:1-106. 
Wang, C. and Aldridge, R. 2010. Silurian conodonts from the Yangtze Platform, South China. Special Papers in Paleontology, 83:1-136.

Witzke, B. 1981. Stratigraphy, depositional environments, and diagenesis of the eastern lowa Silurian sequence. Unpublished PhD Thesis, University of lowa, lowa City, lowa, USA.

Witzke, B. 1992. Silurian stratigraphy and carbonate mound facies of Eastern lowa: Field trip guidebook to Silurian exposures in Jones and Linn Counties, lowa Geological Survey Guidebook Series, 11:3-63.

Won, M., Blodgett, R., and Nestor, V. 2002. Llandoverian (early Silurian) Radiolarians from the Road River Formation of east-central Alaska and the new Family Haplotaeniatumidae. Journal of Paleontology, 76:941-964.

Zhang, S. 2011. Late Ordovician conodont biostratigraphy and redefinition of the age of oil shale intervals on Southamton Island. Canadian Journal of Earth Science, 48:619-643.

Zhang, S. and Barnes, C. 2002. A new Llandovery (Early Silurian) conodont biozonation and conodonts from the Becscie, Merrimack, and Gun River formations,
Anticosti Island, Québec. Journal of Paleontology, 76(suppl. 2):1-46.

Zhang, S. and Barnes, C. 2007a. Late Ordovician to early Silurian conodont faunas from the Kolyma Terrane, Omulev Mountains, northeast Russia, and their paleobiogeographic affinity. Journal of Paleontology, 81:490-512.

Zhang, S. and Barnes, C. 2007b. Late Ordovician-early Silurian conodont biostratigraphy and thermal maturity, Hudson Bay Basin. Bulletin of Canadian Petroleum Geology, 55:179-216.

Zhang, S. and Pell, J. 2014. Conodonts recovered from the carbonate xenoliths in the kimberlites confirm the Paleozoic cover on the Hall Peninsula, Nunavut. Canadian Journal of Earth Sciences, 51:142-155.

Zhen, Y. and Webby, B. 1995. Upper Ordovician conodonts from the Cliefden Caves Limestone Group, central New South Wales, Australia. Courier Forschungsinstitut Seckenberg, 182:265-305.

Zhou, X., Zhai, Z., and Xian, S. 1981. On the Silurian conodont biostratigraphy, new genera and species in Guizhou Province. Oil and Gas Geology, 2:123-140. 


\section{APPENDIX}

Specimen slide SUI numbers.

\begin{tabular}{|c|c|}
\hline \multicolumn{2}{|c|}{ Slide SUI Numbers } \\
\hline Sample & SUI Number \\
\hline GC 365.42-366.25' & 142338 \\
\hline GC 376.5-377.1' & 142339 \\
\hline GC 379.0-379.8' & 142340 \\
\hline GC 387.8-388.5' & 142341 \\
\hline GC 396.58-397.41' & 142342 \\
\hline GC 399.0-399.86' & 142343 \\
\hline GC 409.0-409.86' & 142344 \\
\hline GC 429.1-430.1' & 142345 \\
\hline GC 422.75-423.58' & 142346 \\
\hline GC 427.17-428.09' & 142347 \\
\hline GC 432.92-433.84' & 142348 \\
\hline GC 439.83-440.66' & 142349 \\
\hline GC 445.66-446.53' & 142350 \\
\hline GC 449.87-450.79' & 142351 \\
\hline DX5 0.04-0.08 & 142352 \\
\hline DX5 0.67-0.74 & 142353 \\
\hline DX5 1.34-1.54 & 142354 \\
\hline DX5 3.49-3.59 & 142355 \\
\hline DX5 4.59-4.61 & 142356 \\
\hline
\end{tabular}

